\title{
Ionospheric loss from Mars as predicted by hybrid particle simulations
}

Stephen H. Brecht, Bay Area Research Corp. P.O. Box 366, Orinda CA. 94563 USA

Stephen A. Ledvina, Space Sciences Laboratory, UC Berkeley, 7 Gauss Way, Berkeley, CA, USA

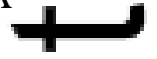

Stephe Bougher, Climate and Space Sciences and Engineering Department, Space Research Buildin niwersity of Michigan, 2455 Hayward St., Ann Arbor, Michigan, 48109-2143 USA
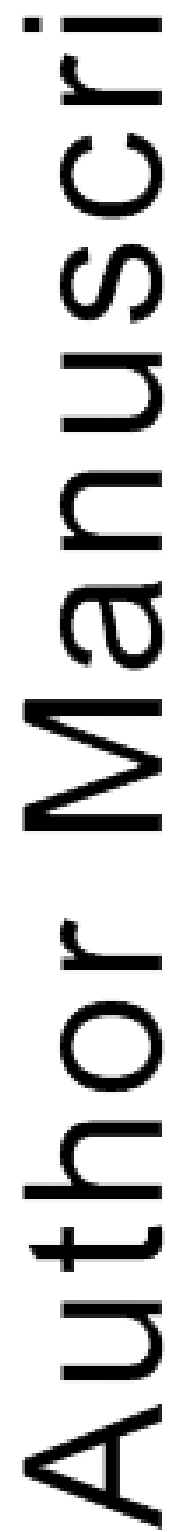

This is the author manuscript accepted for publication and has undergone full peer review but has not been through the copyediting, typesetting, pagination and proofreading process, which may lead to differences between this version and the Version of Record. Please cite this article as doi: $10.1002 / 2016 J A 022548$

This article is protected by copyright. All rights reserved. 
Key Points: Hybrid simulation results are found to match data. The inclusion of 3-D neutral atmospheres with the associated winds causes an increase in the ion loss rate from Mars.

\footnotetext{
(س.

Abstrat. This paper reports the results of hybrid simulations of the Mars-solar wind interaction. The focus is on the loss rates of $\mathrm{O}^{+}$and $\mathrm{O}_{2}^{+}$from the Martian ionosphere while examing (he importance of including 3-D dimensional neutral atmospheres with their associated geutral winds. The simulation loss rates are found to be in excellent agreement with loss rates reported by Lundin et al. [2013]. Further, the results follow the functional form of the loss rates vs. solar EUV as determined by Lundin et al. [2013]. This paper will discuss numerimas as well as the implementation of 3-D neutral atmospheres, including neutral winds ndetation flows.

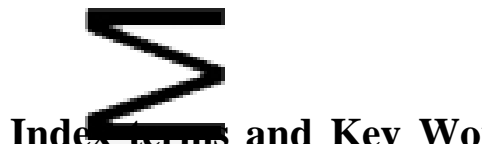

Inde- Trems and Key Words: 0343, 0545, 0654, 2152, 1739, Numerical simulation, Solar wind interaction with Mars, Ionospheric loss from Mars

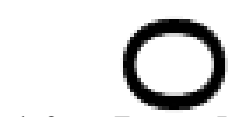

\subsection{Introduction:}

Was Mlars a cold dry planet billions of years ago? Alternatively, was it a wet warm planet? This

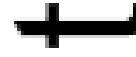

point is under debate. Recent missions to Mars (Curiosity rover) [cf: Martin-Torres et al., 2015 ] and,Mars Reconnaissance Orbiter, MRO, [cf: Ojha, et al., 2015] have found evidence of
} 
water on the Martian surface. Phobos 2, Mars Express (MEX), now MAVEN [cf. Jakosky et al., 2015] have directly measured ionospheric loss of oxygen ions suggesting this as a mechanism for water loss from Mars. The geological evidence suggests that at one time Mars had a substantial amount or water on its surface. The presence of water on Mars and the suggestion that there were suppor life, as we would recognize it? Where did all of the water go? These two related questiofs al driving a significant portion of the Mars exploration.

\section{(S)}

It is thounht that Mars may have had a surface pressure of 1 bar $\left(10^{5} \mathrm{~Pa}\right)$ but loss of the atmosphere has reduced the surface pressure to its present value of 6-10 mbar $\left(10^{2} \mathrm{~Pa}\right)$. One of the momplete papers concerning this topic is by Lammer et al. [2003]. In this paper, the author dSc iss the various mechanisms for water loss. Zahnle [2015] discusses estimates of the waterentory on Mars now and in the past. Estimates of the current water inventory suggest that mertury of the polar ice would cover the Martian surface with up to $30 \mathrm{~m}$ of water. $\mathrm{He}$ reports that the estimates reach approximately $60-90 \mathrm{~m}$ of water 3 billion years ago. Based on $\mathrm{D} / \mathrm{H}$ rad points out that there seems to be a mismatch between the amounts of $\mathrm{H}$ found in the atmosnhere versus $\mathrm{O}$. This apparent mismatch suggests that oxygen escape processes may be different for the hydrogen escape. It is thought that most of the hydrogen escapes via thermal $+$

proces the oxygen escapes via three-body recombination and in ionized form via electromagnetic fields from the solar wind. 
The ion removal process (often called scavenging, stripping, ion escape, ion pickup, etc.) involves a series of interactions leading to the final result: loss of ionospheric ions from Mars to space. incomitration and other solar radiation. This radiation creates an ionosphere by activating a variety of chemical reactions plus photo-ionization. The ionosphere is then subject to time varyin eleg ric and magnetic fields transported by the solar wind through the shock and into the ionospher Strong evidence of the influence of the convection electric field's (measured by Connerneve t al., [2015]) role in ionospheric loss is seen experimentally [cf. Dubinin et al., 2008; Y. Dong, 2014]. The ions respond to these electromagnetic fields by accelerating, eventuming solar wind velocity in the solar wind.

\section{(ర)}

The hrarid particle code, HALFSHEL, simulations of Mars have over the years continued to increasementexity and resolution and are now providing loss rates that are consistent with the dat being reported. Figure 1 is taken from Lundin et al. [2013] and shows a compendium of loss rat $5 y$ a range of years and missions; Phobos- 2 and MEX. The loss rates were estimated based on measurements of ion fluxes with energies between $10 \mathrm{eV}$ and $300 \mathrm{eV}$ to make them consistent across instruments. The loss rates predicted by HALFSHEL for this energy range $+$

(red) thed with results published by Lundin et al. [2013]. One sees very good agreement with regard to the value of the loss rates and the functional dependence on solar EUV flux. This 
comparison indicates that the simulations have reached a sufficient level of complexity and resolution to permit exploration into parameter spaces representative of earlier epochs of Mars. Note, there was no effort made to match the data. Inputs for the simulations were taken from previots MIartian studies found in the literature [cf. Brecht and Ledvina, 2012 and references therein simulations shown and discussed in this paper were performed to examine parameter variations only one of which was the EUV. It should be clear that we could have selecte somr wind variables, crustal magnetic field orientations, Ls, etc. to better match the curves, by hat was not done.

The remander of the paper will provide the reader insight into how these simulations were performand what improvements in the models were required. It will further elucidate the results red by Brecht and Ledvina [2014]. In the next section, a discussion of the HALPSEL hybrid code is presented followed by a section discussing the implementation of the 3-D metraratmospheres. Section 4 will display and discuss the results obtained as a function of solar FUV flux, crustal magnetic field orientation, and the full loss rates with no energy limits placed 7 ge escaping ions. Section 5 will discuss some numerical tests made to check the implementation of the crustal magnetic fields. Finally, Section 6 will discuss and summarize the

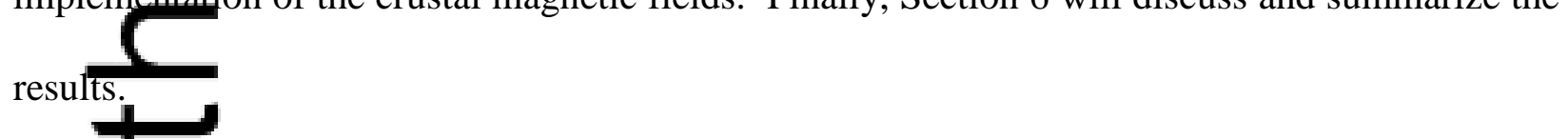




\subsection{Numerical Approach}

Scientists have employed two distinct numerical approaches to estimate the global loss of oxygentrom Mars. One is the MHD formalism [cf: Liu et al., 2001; Ma et al., 2002; Ma et al., 2004; Nagnar, 2007; Harnett and Winglee, 2006; C. Dong et al., 2014]. The second is the kineticformalism, specifically the hybrid particle code [cf: Harned, 1982; Brecht and Thomas, 1988; Crecy, 1990; Brecht and Ferrante, 1991; Kallio and Janhunen, 2002; Bößwetter et al., 2004; Mo ot al., 2005; Chanteur, et al., 2009 ; Holmstr

ditafrod Matition to these two fundamental approaches an ion tracking approach using MHD electromagnetic fields has also been employed [cf: Cravens et al, 2002; Fang et al., 2010 a,b; Curry et al., 2014]. The ion trapproach is an attempt to capture some of the kinetic behavior not found in the MHD OC. This method has the disadvantage that the particle motion and fields are not selfconsistont. A detailed discussion of the equations and assumptions applied in these approaches is contamentrimLedvina at al., [2008].

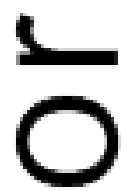

The hybridmodel treats all ion species (the model can carry as many as one wishes) as kinetic particles. A simple Lorentz force equation is used to advance the particle positions and $+1$

velociticlectrons are treated as a massless charge neutralizing fluid. The electromagnetic fields are calculated self-consistently from the ion motion (current) using Ampère's law and a 
generalized Ohm's law. Electromagnetic plasma waves with frequencies up to and including a portion of the whistler wave spectrum are self-consistently produced in the code. Collisionless shock formation physics is included in this code therefore no assumptions or numerical techniques are needed to capture the shock.

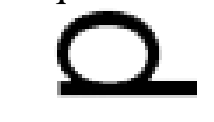

The bacic hybrid model equations are:

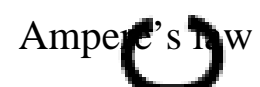

$$
\text { (2) } \nabla \times \mathbf{B}=(4 \pi / \mathrm{c}) \mathbf{J}
$$
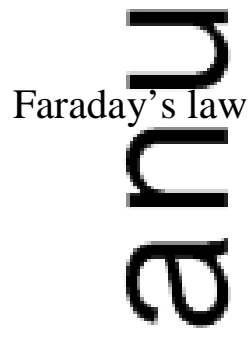

$$
\mathrm{c} \nabla \mathrm{x} \mathbf{E}=-\partial \mathbf{B} / \partial \mathrm{t}
$$

the ion prosicle equations of motion

$$
\mathrm{m}_{\mathrm{i}} \mathrm{d} \mathbf{v}_{\mathrm{i}} / \mathrm{dt}=\mathrm{q}_{\mathrm{i}} \mathbf{E}+\mathrm{q}_{\mathrm{i}} \mathbf{v}_{\mathrm{i}} \mathrm{x}\left(\mathbf{B}+\mathbf{B}_{\mathbf{0}}\right) / \mathrm{c}-\mathrm{q}_{\mathrm{i}} \eta \mathbf{J}
$$
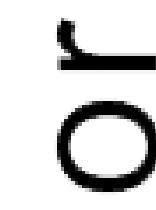

$$
\mathrm{d} \mathbf{x}_{\mathrm{i}} / \mathrm{dt}=\mathbf{v}_{\mathrm{i}}
$$

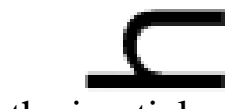

the inertiales electron momentum equation

$$
\longrightarrow_{\text {where }} 0=-\mathrm{e} \mathrm{n}_{\mathrm{e}} \mathbf{E}+\mathbf{J}_{\mathrm{e}} \times \mathbf{B} / \mathrm{c}-\nabla \mathrm{J}_{\mathrm{e}}+\mathrm{e} \mathrm{n}_{\mathrm{e}} \eta \mathbf{J}
$$


and the requirement of quasi-neutrality

$$
\mathrm{n}_{\mathrm{e}}=\mathrm{n}_{\mathrm{i}}
$$

The ele tromperature is evolved by solving the following equation

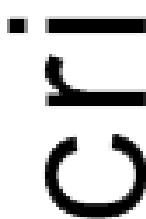

$$
\partial \mathrm{T}_{\mathrm{e}} / \partial \mathrm{t}=-\mathbf{u}_{\mathrm{e}} \bullet \nabla \mathrm{T}_{\mathrm{e}}-3 / 2 \mathrm{~T}_{\mathrm{e}} \nabla \bullet \mathbf{u}_{\mathrm{e}}+2 / 3 \eta \mathbf{J}^{2} / \mathrm{n}_{\mathrm{e}}
$$

where $\mathbf{e}$, electron fluid flow velocity and $\eta$ is the plasma resistivity.

Use of equation 5 allows direct calculation of the electric field via the

Use of equation 5 allows direct calculation of the electric field via the electron momentum equations. This equation also points out the need for three different current systems. The total

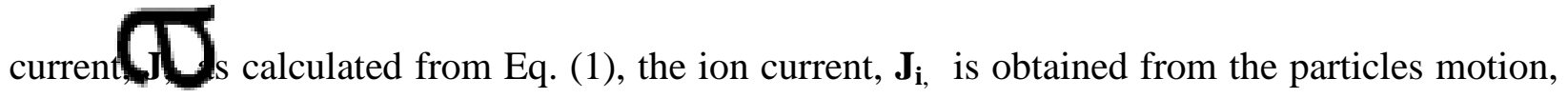
and the $\mathbf{j}$ ctron currents, $\mathbf{J}_{\mathbf{e}}$, obtained by subtracting the ion currents from the total currents. Note that the crustal magnetic fields, $\mathbf{B}_{\mathbf{0}}$, only show up in Eq. (3), the ion equation of motion. The refson for the crustal field only manifesting itself in this one place will be discussed in section of he paper.

Development and use of hybrid particle codes began in the 1970's [cf: Chodura, 1975; Sgro and NEtsoy 1976]. These papers address collisionless shocks and 1-D radial calculations in cylindrical feometry for studying implosion phase of theta pinches. They solved an energy 
equation for the electron heating and used an anomalous resistivity model to explain the large amount of electron heating observed.

In 198 s nybrid code became a common tool for large scale kinetic simulations. Hewett et al. [1980] herf rmed 2-D (r-z) simulations of theta pinch implosions. Harned, [1982] developed an energyconserving predictor-corrector method. This method was used for the first $21 / 2$ - D shock simula Cns Brecht and Thomas, 1988; Thomas and Brecht, 1986a,b, 1987, 1988] and the first 3-D hyprimulations of an AMPTE release [Brecht and Thomas, 1987]. During this time frame many 1-D shock simulations were performed [cf. Winske and Quest, 1988; Quest, 1989]. Brecht, [1990] was the first to point out the existence of shock asymmetry due to diamagnetic effects 3-D hybrid code, HALFSHEL, was then used to simulate Venus and Mars without an iondspe [Brecht and Ferrante, 1991 and Brecht et al., 1993].

The presem version of the hybrid particle code, HALFSHEL [cf. Brecht and Ledvina, 2006, 2010, 2012, 2014 and references therein], was used for this research. The simulations used the approp ato 3-D neutral atmosphere model results from the University of Michigan Mars Thermosnheric General Circulation Model (MTGCM) [cf: Bougher et al., 1999a,b, 2000, 2002, 2004, 2006, and 2008]. These models and their implementation are discussed in section 3 of this paper. 


\subsection{Models operating with HALFSHEL}

Accurate simulation of the solar wind interaction with the Martian ionosphere requires a suite of modelsior important processes. In the next subsections, these models are briefly discussed.

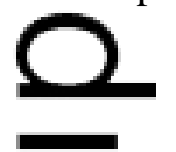

\subsubsection{The photochemistry model}

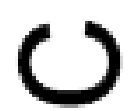

The phropemical chemistry model produces and maintains the ionosphere in the simulations. The ion-neutral chemical reactions (photochemistry) used in HALFSHEL are available in Brecht and Ledvina, [2012]. The reaction set is similar to those reported by [Liu et al., 2001; Ma et al., 2002, The chemistry equations are solved on a high resolution spherical grid surrounding the plabe resith radial resolution of $10 \mathrm{~km}$ and $1.4^{\circ}$ angular resolution. Each numerical particle has a cinsity associated with it and the density is modified by the ion-neutral chemistry using the comenten densities from particles within a spherical grid cell. New particles are created if the ion production rate exceeds the loss rates within each spherical cell. Otherwise, existing particle Iraye their density decreased by chemical processes. Unlike a fluid code, a particle code uses an Euler-Lagrangian approach. Therefore, a particle code can include multiple overlaid grids for differing purposes because the particle location and velocities are independent of a grid. r

\subsubsection{Thecollisional model

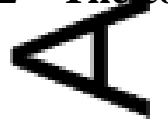


The collisional model addresses species by species collisions using the high resolution grid. The HALFSHEL code treats the ion drag by individually colliding the $\mathrm{H}^{+}, \mathrm{O}^{+}$, and $\mathrm{O}_{2}^{+}$particles with $\mathrm{CO}_{2}$ ando using their respective collision cross-sections. Currently, heating of the particles, (randorizon of their trajectories) is not included. The interaction is simply an energy loss (drag) from the moving particles. This is a "conservative" approach for examining ion loss becaus it oyer estimates the energy deposited in the neutrals but underestimates the ion energy. The neytrofiles are untouched, thus the energy transfer to the neutrals is not accounted for in the simulations, although the ions energy can be augmented via neutral winds. The electron-ion collision frequencies are calculated from Spitzer [1962]. The electron-oxygen (neutral) and electros (neutral) collision frequencies were taken from Strangeway [1996]. The ionneutral colii) frequencies were taken from the expressions in Shunk \&Nagy [2000] using the neutrarpelarizability (Table 1).

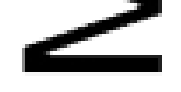

\subsubsection{The conductivity model

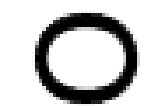

Hall and Pedersen conductivities are included in the electric field solutions. The generalized electric field including the Hall and Pedersen terms comes from a text by Mitchner and Kruger 7 -

[1973] eplaces equation 5 with a more complex form to encompass the conductivity tensor. The generalized electric field [Mitchner and Kruger, 1973] is: 


$$
\boldsymbol{E}=\left(\boldsymbol{J}+\beta_{e} \boldsymbol{J} \times \boldsymbol{B}+s\left(\boldsymbol{B}+\boldsymbol{B}_{0}\right) \times\left(\boldsymbol{J} \times\left(\boldsymbol{B}+\boldsymbol{B}_{0}\right)\right) / \sigma-\nabla \mathrm{p}_{\mathrm{e}} /\left(\mathrm{en}_{\mathrm{e}}\right)\right.
$$

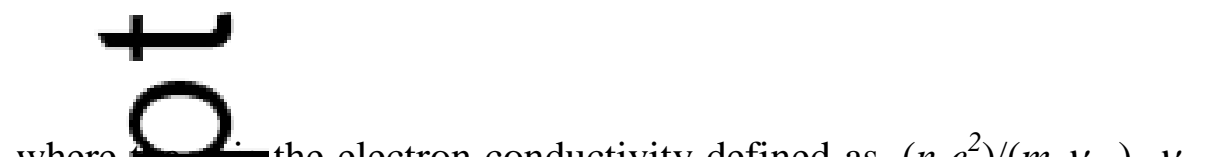

where the electron conductivity defined as, $\left(n_{e} e^{2}\right) /\left(m_{e} v_{e H}\right), v_{e H}$ are the electron collision - -

frequelcies (electron-neutral and electron-ion), $\boldsymbol{J}$ is the current density, $\beta_{e}$ is the electron Hall parame $\left.\operatorname{ter}, \omega_{\mathrm{en}} / v_{\mathrm{eH}}\right)$ with $\omega_{\mathrm{en}}=\mathrm{eB} / \mathrm{cm}_{\mathrm{e}}$, the electron cyclotron frequency. $\boldsymbol{B}$ is the evolving magne fic figld and $\boldsymbol{B}_{\boldsymbol{0}}$ is the crustal magnetic fields. The "ion slip factor", $s$, is $\left(\left(\rho_{n} / \rho\right)^{2} \beta_{e} \beta_{I}\right)$ where $\overline{\rho_{n} 1 \mathrm{~s}}$ he neutral mass density, $\rho$ is the total mass density (ion + neutral), and $\beta_{I}$ is the ion Hall parreter $\left(\omega_{\text {in }} / v_{\text {in }}\right)$. In the limit that the collision frequencies go to zero, this equation returns to the normal electric field equation, Eq. (5). (U⿻

The plasma resistivity for the electron Hall and Pedersen terms are computed from the local spherical grid quantities of electron density, ion density, neutral density, and magnetic field at each timosep. The ion contribution comes from the ion-neutral collision frequency and the ion cyclotrun fr quency calculated locally at each spherical grid point, and the electron-ion collision frequer cy.

2.1.4 magnetic field model

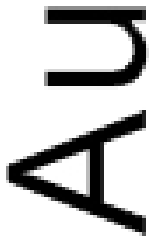


The crustal magnetic field model from Purucker et al. [2000] is embedded in the code. This model returns magnetic field components for altitudes ranging from near surface to beyond $2 R_{m}$. The Martian geological location, the surface longitude and latitude, specifies the crustal magnetic field orrentation. The subsolar point of the crustal field models is specified by the MTC, where MTC Lne airy crater. The code carries the full 3-D crustal field at the - The code includes planetary rotation and inclination, thus the crustal magnef fig ds co-rotate with the neutral atmosphere. Note that the Purucker model or the other crustal fig models available [cf. Cain et al., 2003] are based on data collected at $400 \mathrm{~km}$ by MGS. The accuracy of the models in the subsolar regions is somewhat suspect because the data collected 1s from the night side only. Recall that $1^{\circ}=59.25 \mathrm{~km}$ on the surface and $1.69^{\circ}=100$ $\mathrm{km}$. Mimulations use resolution in this parameter range but it is not clear if the crustal field model arecurate enough at much finer resolution. In section 5 a discussion of this issue will be

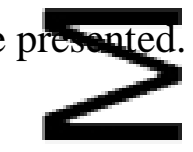

\subsection{5 neutral atmosphere model}

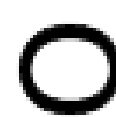

The code-includes a full 3-D neutral atmosphere and associated neutral winds. The neutral atmosphere is the source of the photo-chemically driven ionosphere within the code. Details of $+$

the implation of the neutral atmosphere are found in Sec. 3. The neutral winds modify the ion-neutral drag as well as provide the initial velocity for newly created ions. The neutral 
models used in these simulations are the results of the Mars Thermospheric General Circulation Model, MTGCM, [e.g. Bougher et al., 1988, 1999, 2000, 2004, 2006, and 2008]. A hot O corona based on Chaufray et al. [2007] is also included. Each atmosphere is created with a specific Martial solar longitude and solar fluxes obtained at various points throughout the solar cycle. The so rarsitude, Ls, is the location of Mars in its elliptical orbit about the Sun, where spring equino where $\mathrm{Ls}=0 . \mathrm{Ls}=90$ thus corresponds to summer solstice, just as $\mathrm{Ls}=180$ marks the autumr equinox and Ls=270 the winter solstice (all relative to the northern hemisphere).

\section{(S)}

\subsection{Numerical tests}

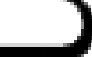

The HaHEL code and its DoD/DoE version have been tested extensively over the years. During the 990 's the HALFSHEL code simulated a variety of experiments. One of them was a laser experiment where structure seen in the experiment was compared to hybrid simuratwors [Brecht and Gladd, 1992]. A fast opening plasma switch was simulated during this time pqriod. Not only was the magnetic data from the experiment mimicked by the simulations, the simaratipns provided the key understanding into the physics of this switches opening; the $\mathrm{m}=1$ kinkinstability.

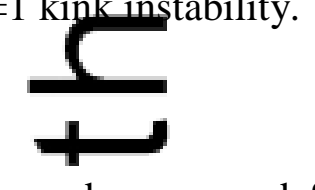

The com shed for simulating shocks where the results were compared to the results of spacecraft measurements of shock encounters. It is important to capture the shock physics 
correctly because the Martian shock sits within the exosphere and very near the ionosphere. One of the data sets for shock comparison was the Voyager 2 encounter with Uranus [Bagenal et al. 1987]. In the mid-1990's, such a test was performed to illustrate the kinetic behavior (overshoot regions and waves) of a high Alfvén Mach shock and check models such as the electron

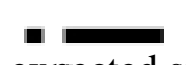
the expected strong overshoot region that reaches a level of roughly 20 [Russell et al, 1982].

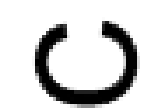

HALFYHF results were compared to the ion density, the magnetic field, and the electron temperature as reported by Bagenal et al. [1987]. Figure 2 shows the magnetic structure of a portion of the Uranian shock as simulated by HALFSHEL. Several features are of note. One, the ovat region is at the appropriate level with large Alfvén Ion Cyclotron, AIC, wave propagtinalong the shock surface [cf: Ambrosiano and Brecht [1987]. Two, behind the shock oversnet one sees the elevated magnetic field with considerable wave activity. Also due to the nature AIC wave, the density and magnetic field oscillation are out of phase. Figure 3 shows the data from Voyager 2 as reported by Bagenal et al. [1987]. The term R-H on the plot by Bag nat et al. [1987] is the Rankine-Hugoniot solution and is used for comparison to the results actually measured. The solid thick line on each of the plots is the result of the simulations averaged along the shock front direction. It is clear choosing an optimal path through the shock $+7$

would the data even better. However, it was felt that comparison to the average would produce the least complex comparison. Comparison of electron temperature, the ion density, and 
the magnetic field shows that the simulation results are in good agreement with the data and verified the general accuracy of the code; particularly of the solution of the electron temperature equation, Eq. 7. This comparison proved that not only was the plasma electrodynamics of a collisiontess shock well represented by the HALFSHEL formulation, but the electron heating equationdunced credible results. This is important for Mars where the shock is very close to the iorosphere and regions where impact ionization and collisions are sensitive to electron temperfure. One might question: Why not perform such a simulation of the Martian shock? The ressg is that until MAVEN missions to Mars did not sample the subsolar region, and carriedeither a magnetometer or an ion plasma instrument, not both.

More the HALFSHEL code has been used to simulate a laser target experiment being perforred the large plasma device, LAPD, at UCLA [Larson et al., 2014] where the wave propaytion speeds measured and simulated agreed to a very high degree.

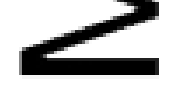

The Cartesian grid resolution used for the Martian simulations has been tested. Changing the grid frg $10 \mathrm{~km}$ to $100 \mathrm{~km}$ resolution produced the same loss rates. Other features changed slightly but_the loss rate remained the same. This is lack of sensitivity to the Cartesian grid resolution occurs because the chemistry is performed on a much finer grid (the spherical grid) I

and the production rates calculated on this finer grid (10 $\mathrm{km}$ radial resolution). It should also be noted that in a particle code the fields are linearly 
interpolated from the cell corners to the particles, thus particles within a Cartesian cell are not accelerated with the same force. The spherical grid overlays the Cartesian grid; particles quantities (density, current, charge) are accumulated on either grid as needed.

The cherkind verification of the code continues and nowhere is this more important than in checking the photochemistry model. The results [Brecht and Ledvina, 2012] match Viking data [e.g. HChsey et al. 1977]. However, it was found that to achieve accurate answers one needed to

The firstecheck on the chemistry determined the time step, $\Delta \mathrm{t}$, needed for an accurate resolution of the chemistry equations. Fig. 4 shows the ratio of highly converged solution to other solutions production of integration time step. The error is computed against the converged solutio 1 si g $\Delta t=0.002 \mathrm{~s}$. When the ratio goes to zero, as is the case for the $\mathrm{O}^{+}$(solid line), often calculated density has gone negative meaning that the solution to the equations has gone turntavle. The electron density, $\mathrm{n}_{\mathrm{e}}$, is the summation of all of the ion species and thus represents the overall error. Fig. 4a displays the solution for neutral densities at $150 \mathrm{~km}$ altitude during ora maximum. One needs to use an integration time step of roughly $0.2 \mathrm{~s}$ or less for accurate_convergence. A more conservative time step of $0.05 \mathrm{~s}$ is used in the full simulations. As the altitude increases, the reactions slow down. Fig. $4 \mathrm{~b}$ shows the ratios for a solar maximum 7

case at ltitude of $200 \mathrm{~km}$. The solutions go unstable at $\Delta \mathrm{t}=13 \mathrm{~s}$. It was found and reported earlier [cf.Brecht and Ledvina, 2012] that one needs to integrate the chemistry out roughly 
$20,000 \mathrm{~s}$ real time before photo-chemical equilibrium is achieved at $400 \mathrm{~km}$ altitude and the plasma dynamics can be started in the simulation. Results from the simulation and testing show that the advection of the plasma is often faster than the photochemical production, thus the two processes are not in equilibrium. This is particularly true at altitudes near or above $250 \mathrm{~km}$ altitud a imulations start out in photochemical equilibrium to an altitude of $400 \mathrm{~km}$, the plasmadynamics then start up and erode the ionosphere until the plasma advection is balanced by the 1 poduction resulting in the final ionospheric density profile.

\section{()}

Additionaltests were performed to check implementation of the chemistry in HALFSHEL. The error here is not one of solution of the coupled set of chemistry equations. It is a test of solving for los and production rates on the spherical grid, interpolating them to particles within the spheriol gy d cell, then interpolating the density back to the grid and solving the chemistry equatrontil there is convergence. A comparable test for a fluid or MHD code would entail solvingemistry on differing grid sizes and then comparing the error in the derivatives of the dersity as required by fluid equations.

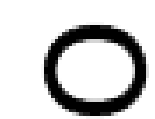

Figure 5-shows the results of performing sensitivity tests as one changed the size of the radial zone, $\Delta \mathrm{r}$, used to integrate the chemistry. In this case we addressed the altitude of $200 \mathrm{~km}$. Here $+$

one se an error of less than $1 \%$ is made using a $\Delta \mathrm{r}=10 \mathrm{~km}$ and $\Delta \mathrm{t}=0.002 \mathrm{~s}$. The error is 
stronger for the $\mathrm{O}_{2}{ }^{+}$and can reach unacceptable levels if the chemistry is not resolved to $20 \mathrm{~km}$ or less.

In the simulations reported in this paper each new atmosphere required that the time step and spatial tep be performed before the simulations were begun. In the next section, we discuss the neutral atmospheres used in these simulations.

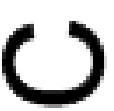

3. The Negral Atmosphere Model.

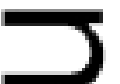

Past research has determined that the chemistry and its response to advection is a crucial part of unders the ionospheric dynamics and the subsequent loss rates from Mars [Brecht and Ledvin 201. In the research reported here a full 3-D neutral atmosphere has been placed withim hybrid code, HALFSHEL. The HALFSHEL code has the ability to make use of the full thmsional neutral dynamics and couple it to the ionosphere dynamics. However, the coupling is only one way at this point with the neutral density and flows not being affected by the ion spmeric dynamics. The neutral atmospheres used here were produced by the MTGCM code. The_density profiles have been extrapolated above the nominal altitude $(250 \mathrm{~km})$ used as an upper boundary for the MTGCM code.

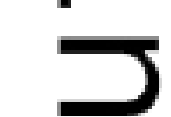

\subsection{A BriefDescription of the Neutral Model.

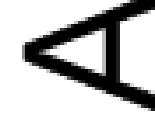


The MTGCM is a finite difference primitive equation model that self-consistently solves for time-dependent neutral temperatures, neutral-ion densities, and three component neutral winds over the inars globe (e.g., Bougher et al., 1999a,b, 2000, 2002, 2004, 2006, and 2008; Bell et al., 2007).

1

The MGG M is driven from below by the NASA Ames Mars MGCM code [Haberle et al., 1999, 2003 at the 1.32- $\mu$ bar level (near 60-80 km). This detailed coupling allows both the migrating and non-migrating tides to cross the MTGCM lower boundary and the effects of the expansion and contraction of the Mars lower atmosphere (due to dust heating) to extend to the thermo The impacts of lower atmosphere dynamics upon the upper atmosphere are domin an most important for reproducing observed thermospheric densities and temperatures [see sorfet al., 2007].

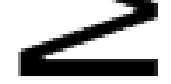

The specific details of the model used are as follows: MTGCM output is for Equinox (Ls = $\left.180^{\circ}\right)$ alar maximum $\left(\mathrm{F}_{10.7}=200\right)$ conditions and $\mathrm{Ls}=270^{\circ}$ and solar median activity $\left(\mathrm{F}_{10.7}\right.$ =130). The $F_{10.7}$ is a proxy for the solar EUV flux at Earth. The actual EUV fluxes represented by this proxy are then adjusted for Mars. The heliocentric distance provides a $1 / \mathrm{R}^{2.0}$ scaling of solar $\mathrm{E}$ V fluxes at Mars for this season. In addition to the neutral densities for $\mathrm{CO}_{2}$ and $\mathrm{O}$ up to $250 \mathrm{~km}$, the neutral models also have the velocity fields for the neutral species. These 
fields are provided in the co-rotational frame of Mars. As will be discussed later, to work in the HALFSHEL code the co-rotational velocities must be included.

Figure snows a typical set of neutral atmosphere parameters produced at $200 \mathrm{~km}$ when the proxy 130. The plots are latitude versus local time. Fig. 6a shows the temperature with horizontal velocity vectors superimposed. Fig. $6 \mathrm{~b}$ shows the $\log 10$ of the $\mathrm{CO}_{2}$ density and Fig. 6c shors the $\log 10$ of the $\mathrm{O}$ density. As can be seen in all three of these plots the flows and densitie not symmetric about the subsolar point. One of the more interesting features is that there seems to be a large amount of $\mathrm{O}$ accumulated on the night side of the planet. This increase in the Odensity will affect the drag on ions transiting the night side but since there is little ionizat night side it will not substantially affect the night side ionospheric production. Furthe $\mathrm{CO}_{2}$ has its highest densities in the subsolar region, while the $\mathrm{O}$ has its highest densitron the night side; showing that the assumption of a symmetric homogeneous neutral atmospreis not correct. This structure coupled with the structure of the crustal magnetic fields means that the solar wind interaction will be more complex than one might have originally envisio
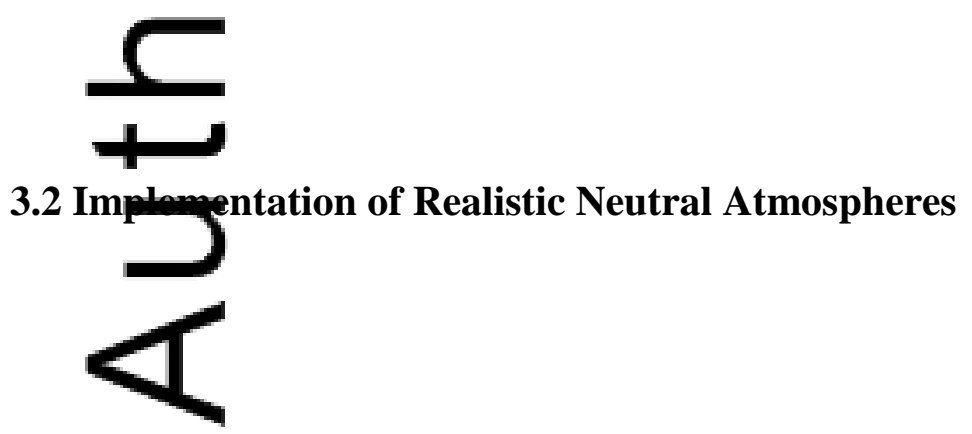
The data from the MTGCM fits easily within the HALFSHEL code. However, a variety of issues had to be addressed. In the following subsection these issues and subsequent tests will be discussed.

\subsubsection{Extrapolation to higher altitudes}

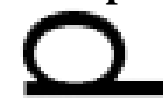

The MTGCM vertical coordinate is in pressure coordinates and has an upper altitude of $\sim 250$ $\mathrm{km}$. The goordinates are Lagrangian and thus move. The hybrid code uses a spherical grid for chemistrand drag calculations. The MTGCM results are provided in spherical coordinates. Therefore the neutral atmospheres and velocity fields fit seamlessly into the HALFSHEL code. The spherical chemistry grid has a comparable radial resolution and a finer angular resolution than thral models.

\section{চ}

Howe there were "holes" where the MTGCM top pressure level sank below the nominal 250 $\mathrm{km}$ arture urface. Furthermore, the neutral density must be extended to much higher altitudes. Diffusine equilibrium was used to extend the neutral grid to $1000 \mathrm{~km}$. The diffusive equilibrium approag tested by taking the neutral atmospheres up to $150 \mathrm{~km}$ and then comparing the diffusive_eauilibrium results with the outputs from MTGCM. As can be seen in Fig. 7, the diffusive equilibrium approximation seems to be valid. The solid curve is the extrapolation from $+1$

$150 \mathrm{kn} 50 \mathrm{~km}$ and the symbols are the MTGCM values The match means that within the 
MTGCM code region the extrapolated results for higher altitude will be a smooth continuation from the MTGCM results.

While tintusive equilibrium is probably not a great assumption, the following rational was used in decinopply this approximation.

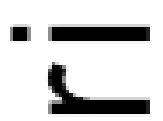

The tratitynal exobase being located around $(\sim 200 \pm 20 \mathrm{~km})$ is not the most recent understang of the transition region between collisional and collisionless regions in the Martian_atmosphere. Detailed DSMC kinetics models [Valeille et al 2010a,b; Lee et al. 2015] reveal that the transition from a collisionally dominated region to a completely kinetic region (e.g. formal O) seems to happen over $200-300 \mathrm{~km}$ (topside depending on Mars season). The MTGCAfl id assumptions are for a completely collisional region, which also transitions over this ange. It is reasonable in this transition region to assume diffusive equilibrium, since ansition to the exosphere (kinetic environment) is gradual ( 200-300 km) and not sharp (t a fixed altitude near $200 \mathrm{~km}$ ).

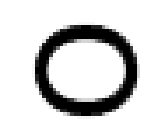

For the HALFSHEL plasma code most of the chemistry occurs at or below $300 \mathrm{~km}$. However, there 1s still photoionization occurring at higher altitudes requiring a reasonable representation of the neumosphere at these higher altitudes. The neutral atmosphere is extended upward from $250 \mathrm{~km}$ using the assumption of diffusive equilibrium so ionization regions have no sharp 
discontinuities that can induce electric fields and motion where none should be found. In short, the goal is to avoid introducing artificial sources of energy by having a gradual and natural transition between collisional and non-collisional regions and this assumption meets the criterion while teing "reasonable." Finally, most of the ion removal is occurring in the region between $200 \mathrm{kmand} b_{50} \mathrm{~km}$ altitude which is in applicable range of MTGCM and thus no assumption of diffusice equilibrium is applied meaning whatever the deficiencies of the diffusive equilibrium assump (1on) he loss rates not strongly affected by the assumption.

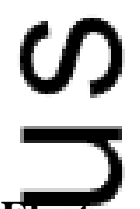

\subsubsection{Electron temperature}

The eldatemperatures from MTGCM were used for a more accurate chemistry calculation as several ot the reaction rates are sensitive to the electron temperature. These temperatures were taken from a standard formulation based upon Viking measurements typically used in 1-D calchrativin (e.g. Fox, 1993). Figure 8 shows the Shinagawa and Cravens [1989] electron temperature profile used in previous simulations and that used from the MTGCM model. The MTGC jectron temperature is easily merged into the Shinagawa and Cravens [1989] temperature_model at high altitudes. The electron temperature profile is held constant below 500 $\mathrm{km}$, while Eq. 7, the electron temperature equation, is solved above this altitude. The altitude for the tramition could be moved to a lower altitude, but the decision was made not to modify it. Recall that the electrons are a fluid and much of the physics to accurately calculate the electron 
temperature at lower altitudes is not in the code: Hence, the decision to hold the electron temperature constant consistent with the MTGCM code and Shinigawa and Cravens [1989]. It is worth noting that Ergun et al. [2015] are suggesting that the details of the electron temperature profiletas it applies to the $\nabla \mathrm{p}_{\mathrm{e}}$ term in the electric field equation may lead to changes in the ratio of the

\subsubsection{Co-rotation of the neutrals.}

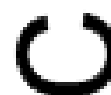

The MTGM neutral models assume the atmosphere co-rotates with the planet. The simulations reportedhare do not make that assumption. Therefore, the co-rotation is added to the neutral flows provided from the MTGCM model. The equatorial co-rotational velocity of Mars ranges from rougnty $245 \mathrm{~m} / \mathrm{s}$ at the surface to slightly more than $280 \mathrm{~m} / \mathrm{s}$ at $500 \mathrm{~km}$. The MTGCM model adictes vertical winds can reach $30 \mathrm{~m} / \mathrm{s}$ while the zonal winds reach velocities $(400 \mathrm{~m} / \mathrm{s})$ which are in excess of the co-rotational velocities. The ions created by photochemical processes are givent the neutral velocity values at their location as provided by the MTGCM neutral atmosplere plus the correction for co-rotation velocity. The presence of the co-rotation velocity will re ace drag on particles being accelerated toward the east but increase it for those going westward With the presence of the neutral flow fields, the imposed E\&M fields from the solar wind, and the rotating crustal fields it is unlikely that the ionosphere is locked into co-rotation but thatringe determined self-consistently. 


\subsubsection{Comments about the 1-D and 3-D neutral atmosphere.}

Research performed using the HALFSHEL code previous to and including Brecht and Ledvina, [2014] used 1-D symmetric fits to the neutral atmosphere. The full 3-D neutral atmosphere is employ f r.the research reported in this paper. Before the results of the simulations are discused, it was felt that a comparison between the 1-D neutral profiles previously used and the 3-D MTGM neutral atmospheres be provided. The most significant comparison simply integratedge entire neutral atmosphere loaded into the HALFSHEL code from $140 \mathrm{~km}$ altitude to $1000 \mathrm{~km}$ altitude. The results were as follows: for the 1-D atmosphere, the total integrated mass was $5.64 \times 10^{10} \mathrm{~kg}$ for the $\mathrm{O}$ and $3.91 \times 10^{12} \mathrm{~kg}$ for the $\mathrm{CO}_{2}$. In the case of the 3-D neutral atmosp results were $4.74 \times 10^{10} \mathrm{~kg}$ for the $\mathrm{O}$ and $1.32 \times 10^{12} \mathrm{~kg}$ for the $\mathrm{CO}_{2}$. The 1-D atmosp aete mad more the mass in $\mathrm{O}$ than the 3-D atmosphere and roughly three (3) times the mass $\mathrm{CO}_{2}$ as the 3-D atmosphere. It will be shown later in the paper that the mass difference resulutim light difference in the ion loss rates.

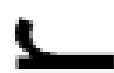

\subsection{Regatts of the simulations}

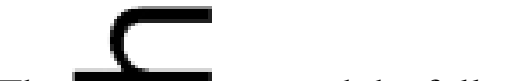

The simulations used the following numerical parameters. The spatial resolution in the Cartesian 12

grid wa $10 \mathrm{~km}$. The spherical grid where chemistry and collisions are calculated employed 10 $\mathrm{km}$ radial resolution and $1.4^{\circ}$ in angular resolution. The time resolution was $0.02 \mathrm{~s}$ for the 
plasma advection and the magnetic field evolution and $0.05 \mathrm{~s}$ for the chemistry equation solutions. This allowed accurate solution of the chemistry equations for all cases; solar minimum to solar maximum. It also allowed accurate solutions over the range of neutral density variations lound in the MTGCM model.

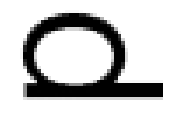

The simulations reported in this paper used a uniform set of solar wind parameters. The solar wind $\mathrm{p}$ amters were as follows: The solar wind speed was $485 \mathrm{~km} / \mathrm{s}$, the IMF was $3 \mathrm{nT}$ with a Parker cipal angle of $56^{\circ}$, and the solar wind density was $2.7 \mathrm{H}^{+} / \mathrm{cm}^{3}$. The rotational axis of the planet is timped at $25.19^{\circ}$ with the orientation determined by the Ls of the simulation. Three different solar EUV levels were used representing solar minimum, a median value commensurate with thar activity at the time of the MAVEN arrival, and solar maximum. As represented by the $F_{10}$ y at Earth, these levels were $F_{10.7}=(70,130,200)$. The EUV levels represented by the Fiongroxy are then adjusted for Mars using $1 / \mathrm{R}^{2}$ scaling to the appropriate location of Mars accorrirg its solar longitude, Ls.

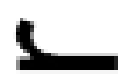

The regits yeflect that the choice of neutral atmosphere affects the loss rates from Mars. In the solar maximum case neutral atmospheres represented by 1-D spherically symmetric models, 3-D models with no winds, and 3-D models with winds were used in simulations. In the solar $+$

maximase with full neutral wind and co-rotation included a series of simulations were performed with the simulations starting at $\mathrm{MTC}$ of $0^{\circ}, 90^{\circ}, 180^{\circ}$, and $270^{\circ}$. In each case, the 
planet was rotating and the simulations were carried out until the loss rate of each ion species reached a steady state $\left(\sim 30\right.$ minutes). Thirty minutes represents only about $7^{\circ}$ of rotation. Ls was $180^{\circ}$ in the solar maximum and minimum cases. In the $\mathrm{F}_{10.7}=130$ case a full 3-D neutral modertitn neutral winds and co-rotation was used. In this case the Ls was $270^{\circ}$ and the MTC was 18

1

The fin at atrator of code behavior and accuracy is comparison to data wherever possible. Fig.

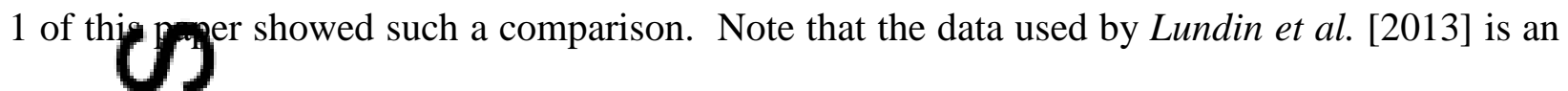
average over solar wind conditions and planetary position in its orbit, Ls. This averaging is one reason why we compare differing Ls and even MTCs. Further, Lundin et al. [2013] also made some 1 assumptions about the homogeneity of ion loss over space in the tail region of Mars. The HAFSHELL simulations very clearly show that the density of ionospheric ions escapng not homogeneous and is in fact time varying; see Fig. 9 and 10. These figures display cuts through the 3-D simulation results for the $\mathrm{O}_{2}{ }^{+}$and $\mathrm{O}^{+}$densities. One cut is in the tailsection of the simulation, the others are in the equatorial plane and the meridional plane. There aype plume region [Dubinin et al., 2008; Y. Dong et al., 2015] where the convection electric field is directed away from the planet. It is clear that the larger gyroradius of the $\mathrm{O}_{2}{ }^{+}$ leads to a more extensive plume while the $\mathrm{O}^{+}$spirals back toward the tail region. It can be stated $+$

with sonfidence that the estimates using the $10 \mathrm{eV}$ to $300 \mathrm{eV}$ energy band is an underestimate (i.e. a wider energy range would capture important lower energy ions that are 
escaping). However, the percentage of the underestimate made by the spacecraft measurements is difficult to assess. The simulation results offer insight into the potential error and will be discussed later, but the error bars associated with the spacecraft measurements are not assessed.

For thenes of this research the ions were considered to have escaped if they passed outward through a virtual box in the simulations set at $2 \mathrm{R}_{\mathrm{M}}$. As each particle passes through this viralahox, its location, velocity, and density weight is recorded. This allows us to not only estimat ion loss, but also assemble distribution functions, higher moments, and time dependent hehavior in any region on the box. It also allows the imposition of energy bands permitting direct comparisons to the experimental results. Thus, discussion of the loss rates will include umergies, and the $10 \mathrm{eV}$ to $300 \mathrm{eV}$ range separately.

\section{(ర)}

\subsection{Losidates as a function neutral atmosphere

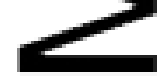

As discussed earlier, simulations were performed with 1-D and 3-D neutral atmospheres. In additio 3 -D atmospheres also had neutral winds included. While the 1-D neutral atmosphere had more mass than the comparable 3-D atmosphere, the 3-D neutral atmosphere with the neutral winds led to greater loss rates. Table 1 offers tabulated results of all of the r.

simulat Fig. 11 shows the results for the $\mathrm{F}_{10.7}=70,130,200$ proxy with neutral winds and co-rotation where available. This choice represents the nominal results although one could have 
chosen results that were even closer to the fit of Lundin et al. [2013]. The circles represent the total loss rate while the stars represent the loss rates in the energy band of $10 \mathrm{eV}$ to $300 \mathrm{eV}$.

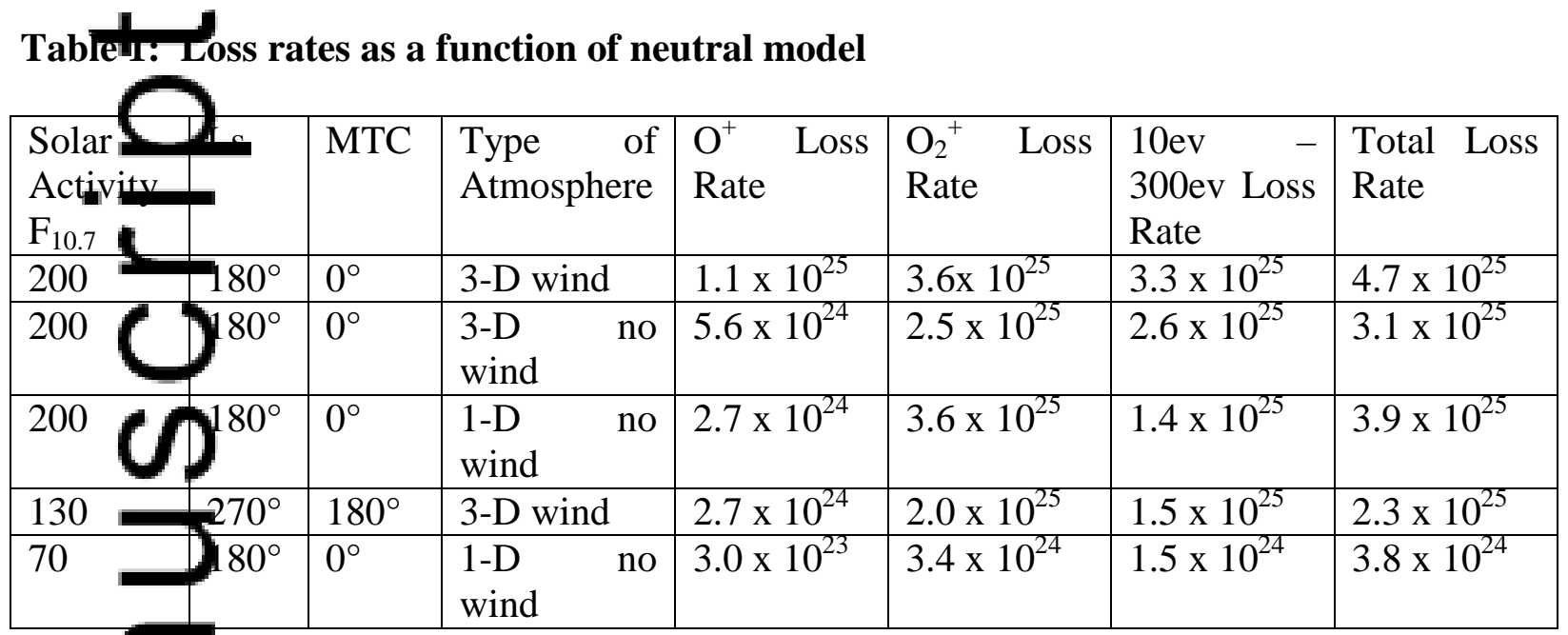

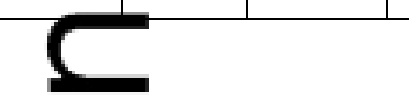

(ে)

The somaximum case was chosen for our comparison between neutral models because the ultimate interest is in conditions where solar EUV fluxes are significantly higher (i.e. a situation expected in earlier Mars epochs). Examining Table 1 one can see that the total loss rates for the 1-D mods lower than the 3-D model with neutral winds but it is higher than the 3-D model withou the neutral winds. Recall that the 1-D model has more mass than the 3-D model.

While D atmosphere had more mass, the 3-D case with neutral winds has more energy. The reated with the neutral wind velocity interact with the crustal magnetic fields to create 
both Lorentz forces and electric fields of sufficient strength to accelerate oxygen ions to escape velocity if the ion remains in the region of the crustal field for a sufficiently long time. It is clear that the inclusion of the 3-D neutral atmosphere with neutral winds produces a result more in keeping with the MEX/ASPERA data. Recall that only the starred values are appropriate for compane Lundin et al. curve. Again, the results are in good agreement with the fit and dat Not too surprisingly, the full loss rates are above the data fit by Lundin et al [2013] as they shoulape. It should be noted that the ratio of $\mathrm{F}_{10.7}$ fluxes are: 1.86 for $\mathrm{F}_{10.7} \mathbf{7 0}$ to 130, 1.54 for $\mathrm{F}_{10} \mathbf{1}^{\mathbf{1 2}}$ to 200, and 2.86 for $\mathrm{F}_{10.7} \mathbf{7 0}$ to 200. However, the ratios of the loss rates are $\sim 6.18$, 2., and 22 for the respective flux ratios. This suggests that the system is not linearly dependent on the EUV although we would argue that solar wind-Mars interaction is a photochemical interac The data, see Fig.1, also indicates this same non-linear behavior.

\section{రে}

\section{2 invore of crustal field orientation

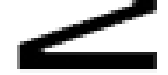

Focusing on the solar maximum case the effect of having different planetary orientations (MTC) facing 1 lar wind was examined. In this situation, the rotating planet covers about $7^{\circ}$ during the time it_takes the ionospheric loss rate to come to steady state. Four simulations were performed each with a different MTC, Fig. 12. The MTC was set to $0^{\circ}, 90^{\circ}, 180^{\circ}$, and $270^{\circ}$. It $+$

should alled that for MTC $=0^{\circ}$ the large crustal fields are at the back of the planet near local midnight. At MTC $=180^{\circ}$ the large crustal magnetic fields are located in the subsolar 
region. Fig. 12 reveals less than a 50\% change in the loss rates from the lowest to the highest loss rates. However, it is interesting to note that the lowest rates occur when MTC is either $0^{\circ}$ or $270^{\circ}$ while the highest is found at $\mathrm{MTC}=90^{\circ}$ and $180^{\circ}$. The average/median rates are close to the fit from Lundin et al. [2013] noting that for our simulations average and median are similar. These are for the solar maximum case where the effect of the crustal magnetic fields is somewhat reduced [Brecht and Ledvina, 2014]. Solar minimum or a more moderate solar activity as yell as a different choice of Ls, might lead to differing results.

\section{$\mathcal{O}$}

\subsection{The relative loss as a function of species}

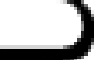

Table some interesting results. The smallest ratio between $\mathrm{O}_{2}^{+}$and $\mathrm{O}^{+}$is found in the solar naxin case that has the neutral winds included. The other solar maximum cases have largen suggesting that the neutral dynamics plays a role in the ratio of the $\mathrm{O}_{2}^{+} / \mathrm{O}^{+}$. The $\mathrm{O}^{+}$ loss fat andignificantly increased as more realistic atmospheres were used.

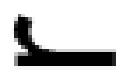

In all of tmy simulations it was found that the $\mathrm{O}_{2}{ }^{+}$was the dominate species lost. However, there are regions_where the $\mathrm{O}^{+}$dominates. Figures 13, 14, and 15 demonstrate these regions. The simulation shown in these figures had a solar EUV index represented by $\mathrm{F}_{10.7}=130$, the MTC 7

was 180 the Ls was $270^{\circ}$; the north pole was tipped away from the sun. In Fig. 13 one sees the evolution of the ratio $\mathrm{O}_{2}{ }^{+} / \mathrm{O}^{+}$as a function of distance down the tail. The regions where the $\mathrm{O}^{+}$ 
flux dominates moves somewhat as the particles make their way out of the Martian system. Figures 14a,b shows the fluxes of $\mathrm{O}_{2}{ }^{+}$and $\mathrm{O}^{+}$at the $2 \mathrm{R}_{\mathrm{M}}$ tailward of Mars. One can see definite structure in both figures with a rather strange triangular shape to the regions. It is clear that overalluthe $\mathrm{O}_{2}{ }^{+}$is the dominate loss species. Examining the ratio of the fluxes, Fig. 14c, reveals that the indeed regions where the $\mathrm{O}^{+}$dominates the loss (blue). Note: the scale is $\log 10$. These egions reside mostly on the side of the planet where the convection electric field points toward he $\mathbf{p}$ lanet. The convection electric field points in the positive $\mathbf{z}$ direction.

\section{(S)}

Fränz et [2010] examined the loss rates measured by MEX at the terminator. Here they found regions where the $\mathrm{O}^{+}$flux dominated the $\mathrm{O}_{2}^{+}$flux. Cuts were made of the simulation through 15 (a) sho $\mathrm{vSth} \mathrm{O}_{2}^{+}$flux and (b) shows the $\mathrm{O}^{+}$. It is clear that once again the $\mathrm{O}_{2}^{+}$dominates just as in the Consistent with Fig. 14c, figure 15c reveals areas in the $-\mathrm{z}$ region of the planet where the muninates. Note: the scale is in $\log 10$. However, one also sees small patches where the $\mathrm{O}^{+}$flux dominates on the dusk side of the planet as well. This implies that orbits will be found yery the measured $\mathrm{O}^{+}$flux will dominate the $\mathrm{O}_{2}^{+}$flux but typically the loss rates will be lower in these regions.

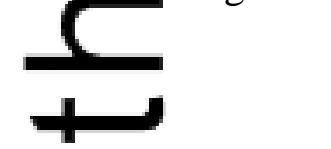

While fluxes appear similar, Fig. 14 and 15, the actual densities are very different. Examination of Fig. 9 and 10 show that the $\mathrm{O}_{2}{ }^{+}$is the dominate density, but it is moving at a 
slower velocity. Hence the flux has regions where the $\mathrm{O}^{+}$dominates. One should also keep in mind that the results shown here include ions of all energies, while the MEX results were limited to ions with energies between $10-300 \mathrm{eV}$.

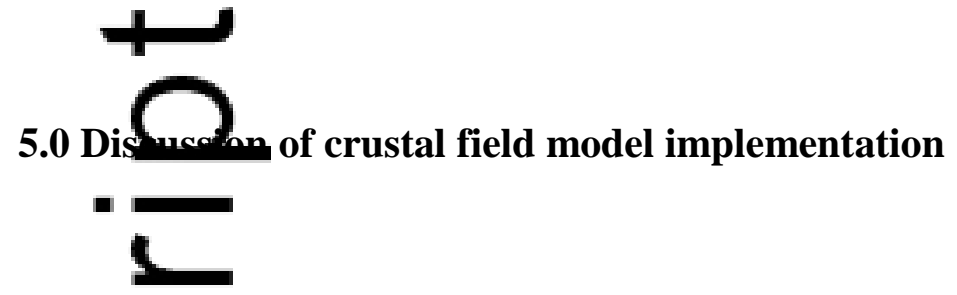

In section 2 it was stated that the crustal magnetic field only entered the calculation via Eq. 3 the particlemation or momentum equation and the conductivity, Eq. 8. This choice was based on both numerical and physical reasoning. The magnetic field models [cf: Purucker et al., 2000; Cain et al., 2003] are high order fits to the MGS magnetometer data taken primarily on the night side at maltitude. As such, the validity of the field models below or above is not very well constrand. This leads to the physical and numerical reasons for caution. On the physical side the reasening is as follows. The sources of the crustal fields are within the surface of the planet, and munaffected by currents set up in the ionosphere. Any measured changes would then be the vector sum of the crustal magnetic fields and the induced fields created by ionospheric current III hort, the crustal magnetic fields themselves will not change. Hence, they cannot be part of eauation (5) which defines the electric field and subsequent magnetic field change via Faraday's law, Eq. 2. The crustal magnetic fields will deflect, rotate, and mirror flowing plasma as it enters them. This will lead to changes in ion and electron currents then changes in electromagnetic fields, and thus modification of plasma behavior. 
The numerical issues are more straightforward. A decision was made that the crustal fields could not be a source of energy; they could not produce current indefinitely (recall the fields themsetves cannot be changed) therefore, $\nabla \times \mathbf{B}_{\mathbf{0}}=\mathbf{0}$ must be maintained. This is accomplished by not the crustal fields in the total current used in electric field equation, Eq. 5. -

Theref re the total current, $\nabla \times \mathbf{B}=(4 \pi / c) \mathbf{J}_{\mathbf{T}}$ only includes the induced current from the IMF. Anoth fea ure that one would like to avoid is the presence of magnetic monopoles, $\nabla \cdot \mathbf{B}_{0} \neq 0$. There 9 eason to suspect or believe that the crustal field models, as complex as they are, will be eitheraty ergence free or curl free especially as implemented onto the code's grid. So again putting Bointo Eq. 5 makes little sense. One could "divergence clean" the fields by computing a vector potential and then a new magnetic field from the vector potential, but then the fields would $D_{b}$ the crustal fields as prescribed by model.

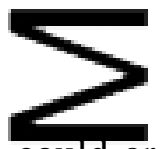

One could argue that the electric field equation should contain a $\mathbf{v}_{\mathbf{e}} \mathbf{x} \mathbf{B}_{\mathbf{0}}$ term. The removal of this terom Eq. 5 is not obvious. Considering Faraday's law, Eq. (2), and substituting in the $\mathbf{v}_{\mathbf{e}} \mathbf{x}$ B term where we use the identities of $\mathbf{J}_{\mathbf{e}}=-\mathbf{J}_{\mathbf{i}}$ and $\boldsymbol{\nabla} \cdot \mathbf{B}_{\mathbf{0}}=0$, one finds terms such as the $\partial \mathbf{B} / \partial \mathrm{t} \propto 2(\mathbf{Y}) \cdot \nabla \mathbf{B}_{\mathbf{0}}+\nabla\left(\mathbf{J}_{\mathrm{i}}\right) \cdot \mathbf{B}_{\mathbf{0}}-\left(\mathbf{B}_{\mathbf{0}} \cdot \nabla\right) \mathbf{J}_{\mathbf{i}}$. 
If one has concerns about the $\nabla \times \mathbf{B}_{0}$ and $\nabla \cdot \mathbf{B}_{0} \neq 0$ terms then one could have some concern about the errors in the $\partial \mathbf{B} / \partial$ t relation. However, it is not obvious that removal of these terms leads to correct answers. If the plasma was simply co-rotating with the crustal fields then the $\mathbf{v}_{\mathbf{e}} \mathbf{X}$ $\mathbf{B}_{\mathbf{0}}$ term would be zero or very small. However, it is clear that the ionospheric flow is not simply co-rotatms of the neutral winds models placed in the code and the effect of the solar wind - -

driven (ields on the upper ionosphere. Several simulations were performed with the $\mathbf{v}_{\mathbf{e}} \mathbf{x} \mathbf{B}_{\mathbf{0}}$ term includ in the code. The simulations included the $F_{10.7}=130$ case and a $F_{10.7}=200$ case. Approf 9 neutral atmospheres were used. The results are tabulated in Table 2.

Table 2. Eoss Rates with $\mathbf{v}_{\mathbf{e}} \mathbf{x} \mathbf{B}_{\mathbf{0}}$ included (U)

\begin{tabular}{|l|l|l|l|l|}
\hline Simulation & Type of Atmos. & $\mathrm{O}^{+}$Loss Rate & $\mathrm{O}_{2}{ }^{+}$Loss Rate & Total Loss Rate \\
\hline $\mathrm{F}_{10.7}=130$ & 3-D wind & $6.0 \times 10^{24}$ & $2.1 \times 10^{25}$ & $2.7 \times 10^{25}$ \\
\hline $\mathrm{F}_{10.7}=\mathbf{0} 00$ & 3-D wind & $8.5 \times 10^{24}$ & $2.1 \times 10^{25}$ & $3.0 \times 10^{25}$ \\
\hline
\end{tabular}

One cat that the loss rates have changed from the rates shown in Fig. 11 and Table 1. This in itself is not bad. However, one notes that the $F_{10.7}=130$ case is now higher than before and the $\mathrm{F}_{10.7}=30$ case is lower. Moreover, the $\mathrm{O}_{2}{ }^{+}$is the same for both cases. While the $\mathrm{O}^{+}$goes up for the $\mathrm{E}_{130}$ case and down for the 200 case. The total loss rates are now very similar. It is 
disturbing that the EUV dependence has been lost. Since data is the final arbitrator of numerical design decisions, it is clear that it was a mistake to include the $\mathbf{v}_{\mathbf{e}} \mathbf{x} \mathbf{B}_{\mathbf{0}}$ term in the simulations. The fears about the implementation of the magnetic crustal field model were well founded.

As a pantretical comment, one notes that in many of the MHD simulations [cf. C. Dong et al., 2014] and some of the hybrid simulations there does not seem to be a strong EUV dependence as seen in he gata. C. Dong et al. [2014] reported loss rates of $2.5 \times 10^{24}$ for solar minimum and 6.6 $\times 10^{24}$ or magnetic fields is causing this lack of sensitivity. We have no knowledge of how these models are implemented in other codes but it is now clear that care must be taken. Finally, one last considauld be addressed. In the situation where there is no solar wind, and the atmosperenosphere has no velocity, the inclusion of the crustal magnetic fields should lead to no moten. As HALFSHEL is configured that is precisely the result obtained.

\subsection{Cogclusions and Discussion}

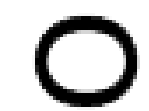

The goal of this research was to address the issue of water loss and specifically the testing and inclusion of improved models necessary for accurate simulation of the loss of $\mathrm{O}^{+}$and $\mathrm{O}_{2}^{+}$. The 1.

compan the results with the fits published by Lundin et al. [2013] strongly suggests that the goal has,been achieved. The research revealed that inclusion of the 3-D neutral atmosphere 
modified the loss rates. The ion loss rates increased to a maximum when the neutral winds and the co-rotation velocity of the neutral atmosphere were present in HALFSHEL. The presence of the neutral winds and co-rotation velocity forced the inclusion of a rotating planet because of the crustămagnetic fields.

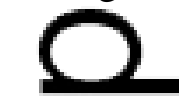

It is beved that the HALFSHEL code is ready to examine earlier epochs to determine if remov of Martian ionosphere by the solar wind is truly a viable water loss mechanism. It should be the loss rate of $10^{26}$ particles $/ \mathrm{cm}^{3}$ will only remove about $1.18 \mathrm{~m}$ of water in $2 \mathrm{GY}$, while it is now estimated that the planet had enough water to cover the entire planet surface in $>40 \mathrm{~m}$ of water. Therefore, the modern loss rates as measure by Phobos-2, Mars Expres now MAVEN [Brain et al., 2015] will not explain the loss by ion removal in earlier epochs temains to be seen if an increase in atmospheric density coupled with higher solar EUV, wher solar wind density, and roughly the same velocity fields will lead to the necessary orde ormatude or two increases in ion escape from Mars. In Brecht and Ledvina [2012] it was found that while the results with the crustal magnetic fields increased with EUV flux, the simulat ons with no crustal fields actually showed a slight decrease in loss rate with increased EUV. As the simulations address earlier epochs (after the magnetic dynamo died $\sim 4$ Gy) with more extensive atmospheres that shield the crustal magnetic fields from the direct interaction $+$

with somind driven fields, will the loss rate still trend upward with increased EUV and solar wind density? 
To compare to the data it was found that using energy bins consistent with the instruments on the spacecraft was crucial and that there is a significant portion of the lost ions at energies below 10 $\mathrm{eV}$ and aoove $300 \mathrm{eV}$. It was also found that increasing the EUV flux as represented by heighted aduction frequencies and differing neutral atmospheres increased the loss rate of ionospheric ions, as one would expect. As explained in Brecht and Ledvina [2014], the crustal magne frylds make a significant difference in the loss rate and as shown in this paper the implemangion of the crustal magnetic field model must be performed with care.

(

During the course of this investigation a variety of tests were performed. The sensitivity of the loss rathe neutral model was tested and it was found that adding the non-isotropic neutral atmospee with neutral winds led to a higher loss rate and better agreement with the data discussed in this paper. It was also shown that careful testing of the integration time step and the spatiar Tesorution of the neutral atmosphere was also very important to obtaining converged accurate solutions.

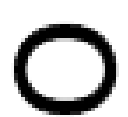

It is clear from the results that changes in the EUV flux is not a direct proxy for the ion loss rates from Mars or even the ion production rates. Mars is a complicated non-linear system were differinatral atmospheres per EUV level, actual EUV photoionization, density dependent ion-neutral photo-chemistry (electron impact ionization, recombination), solar wind driven ion 
dynamics and neutral wind dynamics via ion-neutral collisions all play critical connected roles in determining the ionospheric loss to space.

In sumaresting of each component of the HALFSHEL code has been time consuming but very educational. The simulated global loss rates agree very well with current data. There are isstes romaining to be addressed. One of them is the ratio of $\mathrm{O}_{2}^{+}$to $\mathrm{O}^{+}$. As reported in this paper itfound that $\mathrm{O}_{2}{ }^{+}$dominates the loss. However, earlier MEX data [cf. Nilsson et al, 2011; Carlson et al.,2006] report loss rates where the two species are close to equal. However, Nilsson et al comments "We have used the lowest energy data (up to 50eV) where the mass separatworks the best to estimate the relative amount of each species..." This limited energy range of of allow for a determination of the dominate species. MAVEN data as published by Bennat. [2015] and Ergun et al., [2016] show equal amounts of $\mathrm{O}_{2}{ }^{+}$to $\mathrm{O}^{+}$at altitudes up to 500 mintm is very interesting because simple photo-chemical results show the $\mathrm{O}_{2}{ }^{+}$rapidly dropping to zero above $300 \mathrm{~km}$. Initial comparisons with HALFSHEL results, show very similar results OSenna et al.[2015] and Ergun et al., [2016]. However, the complexity of the comparison_prevents further discussion in this paper. Finally, detailed comparison against MAVEIN data [cf. Jakosky et al, 2015] as it becomes available should add further credence to the simulat ands andor illuminate areas where the modeling must be improved. 


\section{ACKNOWLEDGEMENTS:}

The S.H. Brecht and S.A. Ledvina would like to acknowledge the support from NASA contract NNHTLCF43C. S.W. Bougher was supported in part by NASA MDAP grant NNX12AJ49G throug the computational support provided by the NASA Advanced Supercomputer facility, NAS, at NASA Amys Research Center Moffett field CA. Finally, data from which the results of this paper ame aren available upon contact with SHB or SAL.

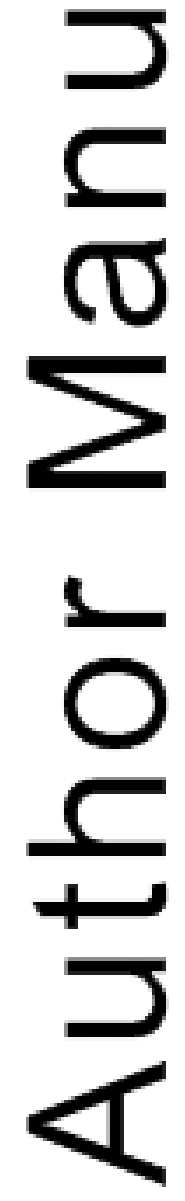




\section{References}

Ambrosiano, J., and S.H. Brecht (1987), "A simulation study of the Alfvén ion-cyclotron instability in high-beta plasma," Phys. Fluids, 30, 108.

Bagenal, F.J., W. Belcher, E.C. Sittler, and R.P. Lepping, (1987), "The Uranian bow shock: Voyagrs 2 i lbound observations of a high Mach number shock," J. Geophsys. Res.,92, 8603.

Bell, J M., W. Bougher, and J. R. Murphy (2007), Vertical dust mixing and the interannual variaticne Mars thermosphere, J. Geophys. Res., 112, E12002, doi:10.1006JE002856.

Benna,'vi.,P.R. Hahaffy, J.M. Grebowsky, J.L. Fox, R.V. Yelle, and B.M Jakosky (2015), “ First mastoments of composition and dynamics of the Martian ionosphere by MAVEN's Neutra Gad and Ion Mass Spectrometer," Geophys. Res. Lett., 42 8958-8965, doi:10.1002/2015GL066146.

Bößwetter, A., Bagdonat, T., Motschmann, U., and Sauer, K, (2004), "Plasma boundaries at Mars: a 3-D simulation study," Annales Geophysicae,22, 4363.

Bougher. S. W., R. E. Dickinson, R. G. Roble and E. C. Ridley (1988), Mars thermospheric genera circulation model - Calculations for the arrival of PHOBOS at Mars, Geophys. Res. Lett., 15, 15 11-1574.

Bough .J. et al. (1999a), Mars Global Surveyor aerobraking: Atmospheric trends and model interpretation, Adv. Space Research, 23, 1887-1897 doi:10.1016/S0273-1177(99)00272-0.

Bougher S. W., S. Engel, R.G. Roble, B. Foster (1999b), Comparative terrestrial plane trermospheres 2. Solar cycle variation of global structure and winds at equinox. J. Geophys. Res. 104, 16591-16611 doi:10.1029/1998JE001019

Bougher, S. W., S. Engel, R.G. Roble, B. Foster (2000), Comparative terrestrial planet thrmospheres 3. Solar cycle variation of global structure and winds at solstices. J. Geophys. Res. 105, 17669-17692 doi:10.1029/1999JE001232

Bough(r, S. W., R.G. Roble, T.J. Fuller-Rowell (2002), Simulations of the upper atmospheres of the terrestrial planets, in Atmospheres in the Solar System, Comparatrve Aeronomy, ed. by M. Mendillo, A.F. Nagy, J.H. Waite Jr. AGU Monogw vol. 130 (American Geophysical Union, Washington, pp.261-288

Bougher, S. W., S. Engel, D.P. Hinson, J.R. Murphy (2004)., MGS Radio Science electrar lensity profiles: Interannual variability and implications for the Martian neutral 
atmosphere, J. Geophys. Res. 109, E03010 doi:10.1029/2003JE002154

Bougher, S. W., J.M. Bell, J.R. Murphy et al. (2006), Polar warming in the Mars thermosphere: Seasonal variations owing to changing isolation and dust distributions. Geophys. Res. Lett. 33, L02203 doi:10.1029/2005GL024059

Bougnter, S. W., P.-L. Blelly, M. Combi et al. (2008), Neutral upper atmosphere and ion sppre modeling. Space Sci. Rev. 139, 107-141 doi:10.1007/s11214-008-9401-9

Bougher et al. (2008), "Neutral Upper Atmosphere and Ionosphere Modeling," Space Sci. Rev.,139, 107-141 doi:10.1007/978-0-387-87825-6_4.

Brain, D. A., J. P. McFadden, J. S. Halekas, J. E. P. Connerney, S. W. Bough 5. Curry, C. F. Dong, Y. Dong, F. Eparvier, X. Fang, et al. (2015), "The spatial distribution of planetary ion fluxes near Mars observ ( by MAVEN," Geophys. Res. Lett., 42,9142-9148, doi:10.1002/2015GL065293.

Brecht_S and V.A. Thomas (1987), "Three-Dimensional Simulation of an Active Magnetospheric Release," J. Geophys. Res., 92, 2289.

Brecht and V.A. Thomas (1988), "Multidimensional simulations using hybrid particle codes," Comm. , 48, 135.

Brecht, S.M. (1990),"Magnetic asymmetries of unmagnetized planets," Geophys. Res. Lett., 17, 1243

Brech.H. and Ferrante, J.R. (1991), "Global Hybrid Simulation of Unmagnetized Planets: Comparison of Venus and Mars," J. Geophys. Res., 96, 11209.

Brecht S.H. and N.T. Gladd, "Structuring of a plasma shell expanding into a magnetized plasma at sub-Alfvenic speed," IEEE Transactions on Plasma Sci., 20, 678.

Brecht, S.H, J.R. Ferrante, and J.G. Luhmann, (1993), "Three-Dimensional Simulations of the Solar Wind Jnteraction With Mars," J. Geophys. Res., 98, 1345.

Brectur., and S.A. Ledvina (2006), "The solar wind interaction with the Martian Ionospera/A tmosphere," Space Sci. Rev., 126, 15.

Brecht, S. I., and S A. Ledvina (2010), "The loss of water from Mars: Numerical results and challengove Icarus, 206, Issue 1, Solar Wind Interactions with Mars, 164-173, ISSN 00191035, DOT0.1016/j.Icarus.2009.04.028. 
Brecht, S. H., and S. A. Ledvina (2012), "Control of ion loss rates from Mars during solar minimum," Earth Planets Space, 64, 165-178.

Brecht, S.H. and S.A. Ledvina (2014), "The role of the Martian crustal magnetic fields in controlling ionospheric loss, “Geophys. Res. Lett., 41, 5340-5346, doi:10.1002/2014GL060841.

Cain Iosepla C.; Ferguson, Bruce B.; Mozzoni, David, 2003, “An n = 90 internal potential function of the Martian crustal magnetic field," J. Geophys. Res. (Planets), 108, DOI 10.102/2000JE001487.

Carlsson, E. et al. (2006)," Mass composition of the escaping plasma at Mars," Icarus, 182, 320-32

Chaufry Y., R. Modolo, F. Leblanc, G. Chanteur, R. E. Johnson, and J. G. Luhmann (2007),"Mars solar wind interaction: Formation of the Martian corona and atmospheric loss to space, J G ophys. Res., 112, Issue E9, CiteID E09009.

Chanteur, M., E. Dubinin, R. Modolo, and M. Fraenz (2009), "Capture of solar wind alphaparticleard the Martian atmosphere," Geophys. Res. Lett., 36, L23105, doi:10.1020/2009GL040235.

Chodura, R., "A hybrid fluid-particle model of ion heating in high-Mach-number shock waves ," Nuclea (usjon, 15, 55, 1975.

Cont J. E. P., J. R. Espley, G. A. DiBraccio, J. R. Gruesbeck, R. J. Oliversen, D. L. Mitchell, J. Halekas, C. Mazelle, D. Brain, and B. M. Jako (2015), First results of the MAVEN magnetic field investigation, Geophys. Res. Lett., 42, 8819-8827, doi:10.1002/2015GL065366.

Craven T_E., A. Hoppe, S. A. Ledvina, and McKenna-Lawlor, S. (2002), "Pickup ions near Mars associated with escaping oxygen atoms," J. Geophys. Res., doi:10.1029/2001JA000125.

Curry, M., M. Liemohn, X. Fang, Y. Ma, J. Slavin, J. Espley, S. Bough and C. F. Dong (2014), Test particle comparison of heavy atomic and distributions at Mars, J. Geophys. Res. Space Physics, 119, 2328_2344, doi:10.1002/2013JA019221.

Dong, C., S. W. Bougher, Y-J Ma, G. Toth, A. F. Nagy, and D. Najib, (2014),"Solar wind interactorrwith Mars upper atmosphere: Results from the one-way coupling between the multifluid MHrodel and the MTGCM model," accepted J. Geophys. Res. 
Dong, Y., X. Fang, D. A. Brain, J. P McFadden, J. S. Halekas, J. E. Connerney, S. M. Curry, Y. Harada, J. G. Luhmann, and B. M. Jakosky (2015), Strong plume fluxes at Mars observed by MAVEN: An important planetary ion escape channel, Geophys. Res. Lett., 42, 8942-8950,doi:10.1002/2015GL065346

Dubi- et al., (2008), "Structure and dynamics of the solar wind/ionosphere interface on Mars: MEX-ASPERA-3 and MEX-MARSIS observations," Geophys. Res. Lett., 35, doi: 10 2029 2008L033730.

Ergun, T.L. et al. (2016), "Enhanced $\mathrm{O}_{2}{ }^{+}$loss at Mars due to ambipolar electric field from electro heating,", J. Geophys. Res. Space Physics, 121, 4668-4678, doi: 10.1002/2016JA022349.

Fang, X.Michael W. Liemohn, Andrew F. Nagy, Janet G. Luhmann, Yingjuan Ma (2010a), "On the effet of the Martian crustal magnetic field on atmospheric erosion," Icarus, 206, Issue 1, Solar Gidd Interactions with Mars, Pages 130-138, ISSN 0019-1035, DOI: 10.1016j-Larus.2009.01.012.

Fang, X., M. W. Liemohn, A. F. Nagy, J. G. Luhmann, and Y. Ma (2010b), "Escape probability of Ma ian atmospheric ions: Controlling effects of the electromagnetic fields," J. Geophys. Res., 1 Tro4308, doi:10.1029/2009JA014929.

Fox, J.4.193), “The Production and Escape of Nitrogen Atoms on Mars", J. Geophy. Res., 98, $3297-3310$

Fränz_Dubinin, E., Nielsen, E., Woch, J., Barabash, S., Lundin, R., Fedorov, A.,2010. Transtermmmator ion flow in the Martian ionosphere. Planet. Space Sci. 58, 1442-1454.

Harned D.S. (1982)," Quasineutral hybrid simulations of macroscopic plasma phenomena," $J$. Comp. Phys., 47, 452.

Harnet E. and Winglee, R. M. (2006), "Three-dimensional multifluid simulations of ionosphanialoss at Mars from nominal solar wind conditions to magnetic cloud events," J. Geoph s. Res., 111, DOI:10.1029/2006JA011724.

Hansoli, W. B., S. Sanatani, and D. R. Zuccaro (1977), "The Martian ionosphere as observet by the Viking retarding potential analyzers," J. Geophys. Res., 82, 4351

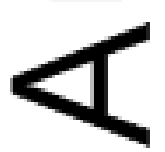


Haberle, R.M., et al., (1999), "General circulation model simulations of the Mars Pathfinder atmosphere investigation/meteorological data," J. Geophys. , 104, 8957, doi:10.1029/1998JE900040.

Haberle, R.M., J.L. Hollingsworth, A. Coaprete, A.F.C. Bridger, C.P. McKay, J.R. Murphy, J. Schaeffer, and R. Freedman (2003), "The NASA/AMES general circulation model: Model improvements and comparison with observations, In Published Conference Abstract, Interng Cony Workshop: Mars Atmosphere Modelling and Observations.

Hewett_D. (1980), J. Comput. Phys., 38, 378.

Holms ominand (5), "Mars as a comet: Solar wind interaction on a large s ale, physics.space-ph, 1.

Jakosky R. M., J. M. Grebowsky, J. G. Luhmann, and D. A. Brain (2015), Initial eslts from the MAVEN mission to Mars, Geophys. Res. Lett., 42, 8791-8802 doi:10.1002/2015GL065271.

Kallio, E., and Janhunen, P. (2002), "Ion escape from Mars in a quasi-neutral hybrid model,", $J$. Geoph S.Res., 107, doi:10.1029/2001JA000090.

Lammer Lichtennegger, H.I.M., Kolb, C., Ribas, I., Guinan, E.F., Abart, R., and. Bauer, S.J, (2003) Co s of water from Mars: Implications for the oxidation of the soil," Icarus, 165, 9, doi:10.1016/S0019-1035(03)00170-2, .

Larson.D. T.S. Islam, and S.H. Brecht (2014), "Three dimensional hybrid simulations of the UCL Com in ionless shock experiment and related high altitude nuclear explosion phenomena," Hardened Electronics and Radiation Technology Technical Interchange Meeting, March 17-21.

Ledvina, S.A., Y.-J. Ma and E. Kallio (2008), "Modeling and simulating flowing plasmas and related Mremena," Space Sci Rev., 139, 143-189, DOI 10.1007/s11214-008-9384-6.

Lee, Y., M. R. Combi, V. Tenishev, S. W. Bougher, J. Deighan, N. M. Schneider, W. E. McCli tock, B. M. Jakosky (2015), "A comparison of 3-D model predictions of Mars' oxygen cororra with early MAVEN IUVS observations," Geophys. Res. Lett., 42, DOI: 10.10م5065291.

Liu, Y., Nayy, A.F., Gombosi, T.I., DeZeeuw, D.L., and Powell, K.G. (2001), "The solar wind interacturnith Mars: Results of the three-dimensional three species MHD studies," Adv. Space Res. 27, $107 \%$. 
Lundin, R, S. Barabash, M. Holström, H. Nilsson, Y. Futaana, R. Ramstad, M. Ymauchi, E. Dubinin, and M. Fraenz (2013), "Solar cycle effects on the ion escape from Mars," Geophy. Res. Lett., 40, 6028-6032, doi:10.1002/2013GL058154.

Ma, Y., Nagy, A.F., Hansen, K.C., and DeZeeuw, D.L. (2002), “Three-dimensional multispecies MHD studies of the solar wind interaction with Mars in the presence of crustal fields, ", $J$. Geopnys. Res., 107, 1282, doi:10.1029/2002JA009293.

Ma, Y Na. A. A. F., Sokolov, I.V., and Hansen, K.C. (2004), "Three-dimensional, multispecies, high spatial resolution MHD studies of the solar wind interaction with Mars," J. Geophys. Res. , 109, 10 1029/2003JA010367.

Martin-Torres et al., (2015), Transient liquid water and water activity at Gale c ater $n$ Mars. Nat. Geosci. http://dx.doi.org/10.1038/NGEO2412.

Ma Y.c.and A.F. Nagy (2007), "Ion escape fluxes from Mars," Geophys. Res. Lett., 34, L0820 1029/2006GL029208.

Mitchner, M. and Kruger (1973), C.H., Jr., Partially Ionized Gases, John Wiley \& Sons, NY, USA.

Modol R. Chanteur, G.M., Dubinin, E., and Matthews, A.P. (2005), "Influence of the solar EUV flux on the Martian plasma environment," Annales Geophysicae, 23, 433.

Nagy, 2004), “The plasma environments of Mars," Space Sci. Rev., 111, Nos. 1-2, 33.

Nilssom. et al., (2011), "Heavy in escape from Mars, influence from solar wind conditions and crustal magnetic fields," Icarus, 215, 475-484, doi:10.1016/j.icarus.2011.08.003.

Ojha, L. et al., 2015. Spectral evidence for hydrated salts in recurring slope lineae on Mars. Nat. Geosci http://dx.doi.org/10.1038/ngeo2546

Purucker_M.; Ravat, D.; Frey, H.; Voorhies, C.; Sabaka, T.; Acuna,M. (2000), "An altitudenormal zed hagnetic map of Mars and its interpretation," Geophys. Res. Lett., 27, 2449.

Quest, 1 (1989), "Hybrid simulations," in Tutorial courses: Third International School for Space Fimulations, B. Lembege and J.W. Eastwood, Eds. Toulouse, France: Cepadues Edition, p. 117.

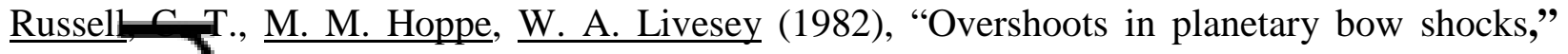
Nature 296 5852):45-48. DOI: 10.1038/296045a0.

Sgro A and C. W. Nielson (1976), "Hybrid model studies of ion dynamics and magnetic field diffusion luring pinch implosions ," Physics of Fluids 19, 126; doi: 10.1063/1.861309 
Spitzer, L. (1962), "Physics of Fully Ionized Gases," $2^{\text {nd }}$ Ed. Interscience Publishers, p 127.

Shinagawa, H. and Cravens, T.E.: 1989, "A One-Dimensional Mult-species

Magnetohydrodynamic Model of the Dayside Ionosphere of Mars," J. Geophys. Res., 94, 6506.

Shunk and Nagy (2000), "Ionospheres," Cambridge Univ. Press, p83, 1st edition

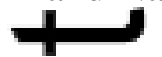

Strangeway, R. (1996),"Collisional Joule Dissipation in the Ionosphere of Venus: The Import nce ff Electron Heat Conduction", J. Geophys. Res. , 101, 2279-2295.

Thoma, V.A. and S.H. Brecht, (1986a) "Two dimensional hybrid simulations of high Mach number prasma interactions," Phys. of Fluids, 29, 2444.

Thoma (V). and S.H. Brecht, (1986b), "Simulation of magnetic phenomena driven by parallel ion motion" Phys. of Fluids, 29, 3398.

Thoma, a. and S.H. Brecht, (1987), "Angular dependence of high Mach number plasma interactions' J. Geophys. Res., 92, 3175.

Thomas, V.A. and S.H. Brecht, (1988), "Evolution of Diamagnetic Cavities in the Solar Wind," J. Geo hys. Res., 93, 11341.

Valeille A M. Combi, V. Tenishev, S. Bougher, and A. Nagy (2010a), "A study of supratl er na oxygen atoms in Mars upper thermosphere and exosphere over the range of limiting conditions," Icarus, 206, 18-27, doi:10.1016/j.icarus.2008.08.018.

Valeille, SW. Bougher, A., V. Tenishev, M. Combi, and A. Nagy (2010b), "Water loss and evol the upper atmosphere and exosphere over Martian history," Icarus, 206, 28-39, doi:10.1016/j.icarus.2009.04.036.

Winsk and K.B. Quest (1988), "Magnetic field and density fluctuations at perpendicular supercritical collisionless shocks," J. Geophys. Res., 93, 9681.

Zahnle, 2015), "Play it again, SAM," Science, 347, Issue 6220, 370.

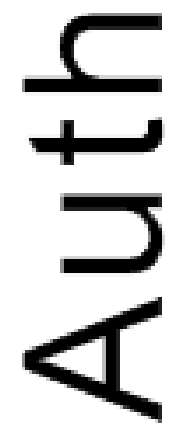




\section{FIGURE CAPTIONS:}

Figure 1: Plots from Lundin et al. [2013] showing both the median escape rates (a) and the average escape (b) rates as a function of EUV ( $\mathrm{F}_{10.7}$ proxy). In red are the HALFSHEL loss rates in the same $10 \mathrm{eV}$ to $300 \mathrm{eV}$ energy range.

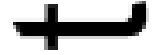

Figure 2-The magnitude of B in Gauss from the 2-1/2 D hybrid simulation of the Uranus bow shock. Note the overshoot region produces a jump of 20 and is highly structured. The inflow conditions were the solar wind conditions reported by Bagenal et al. [1987].

Figure somparison of the simulation results with the data reported by Bagenal et al. [1987] The sold line represents the simulation results. The vertical line is the end of the simulation coverag ote the excellent agreement with the electron temperature as well as with the proton density

Figure 4. (3) The ratio of individual ion species density to a converged solution at $150 \mathrm{~km}$ is shown. (b) The ratio of individual ion species density to a converged solution at $200 \mathrm{~km}$ is shown The converged solution was created using $\Delta \mathrm{t}=0.002 \mathrm{~s}$.

Figure 5 The error in chemistry as a function of the size of $\Delta \mathrm{r}$ in $\mathrm{km}$. The solid line is $\mathrm{O}_{2}{ }^{+}$and the das 1 ne is $\mathrm{O}^{+}$.

Figure Maps of the neutral atmosphere of Mars at $200 \mathrm{~km}$ altitude. The subsolar is at 12 o'clock ant the EUV indicator is $\mathrm{F}_{10.7}=130$. (a) Shows the neutral temperature and velocity vectors minte horizontal plane (the largest vector is $523 \mathrm{~m} / \mathrm{s}$ ). (b) Shows the Log 10 of the $\mathrm{CO}_{2}$ neutral density. (c) Shows the Log10 of the O neutral density.

1

Figure The left plot is of the test with $\mathrm{CO}_{2}$. The right plot is the test with $\mathrm{O}$.

Figure 8 Electron temperature profile as a function of altitude. The continuous line is the fit from Slinagawa and Cravens and the symbols are from the MTGCM model.

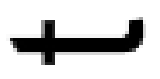

re 9

Figure $\mathrm{O}_{2}{ }^{+}$ions as they escape Mars' ionosphere.

Figure 10. Plot of the $\mathrm{O}^{+}$ions as they escape from Mars' ionosphere. 
Figure 11. Plot of the two scaling relations from Lundin et al. [2013] and the results of various simulations and energy bands. The circles are the total loss rates including all energy bands.

Figure 12. Loss rates as a function of MTC or sub-solar longitude. The full circles are the total loss rates, the stars are the loss rates for the $10 \mathrm{eV}$ to $300 \mathrm{eV}$ energy band. Note that the variation fits better to the median (dashed) fit rather than the mean (solid). The average/median values of the loss rate are slightly higher than the fit. There is less than a 50\% variation from minimum to maximan rate suggesting that the crustal field orientation is not a major factor in determ mind the loss rate.

Figure 3. The ratio of $\mathrm{O}_{2}^{+} / \mathrm{O}^{+}$at the terminator, $2 \mathrm{R}_{\mathrm{M}}$, and $4 \mathrm{R}_{\mathrm{M}}$. The scale is in log10.Figure 14 . The loss mux of both $\mathrm{O}_{2}^{+}$(a) and $\mathrm{O}^{+}$(b) as measured at $2 \mathrm{R}_{\mathrm{M}}$ downstream of Mars. (c) The ratio of flux $s$ for $\mathrm{O}_{2}^{+}$and $\mathrm{O}^{+}$at $2 \mathrm{R}_{\mathrm{M}}$ in the tail.

Figure 15 The anti-sun flux in the $+\mathbf{x}$ direction at the terminator of Mars as seen from the Sun $(\mathrm{a}, \mathrm{b})$. W tio of $\mathrm{O}_{2}^{+} / \mathrm{O}^{+}$is shown in (c).

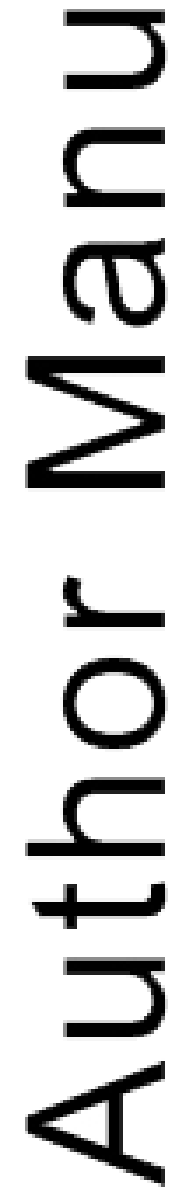



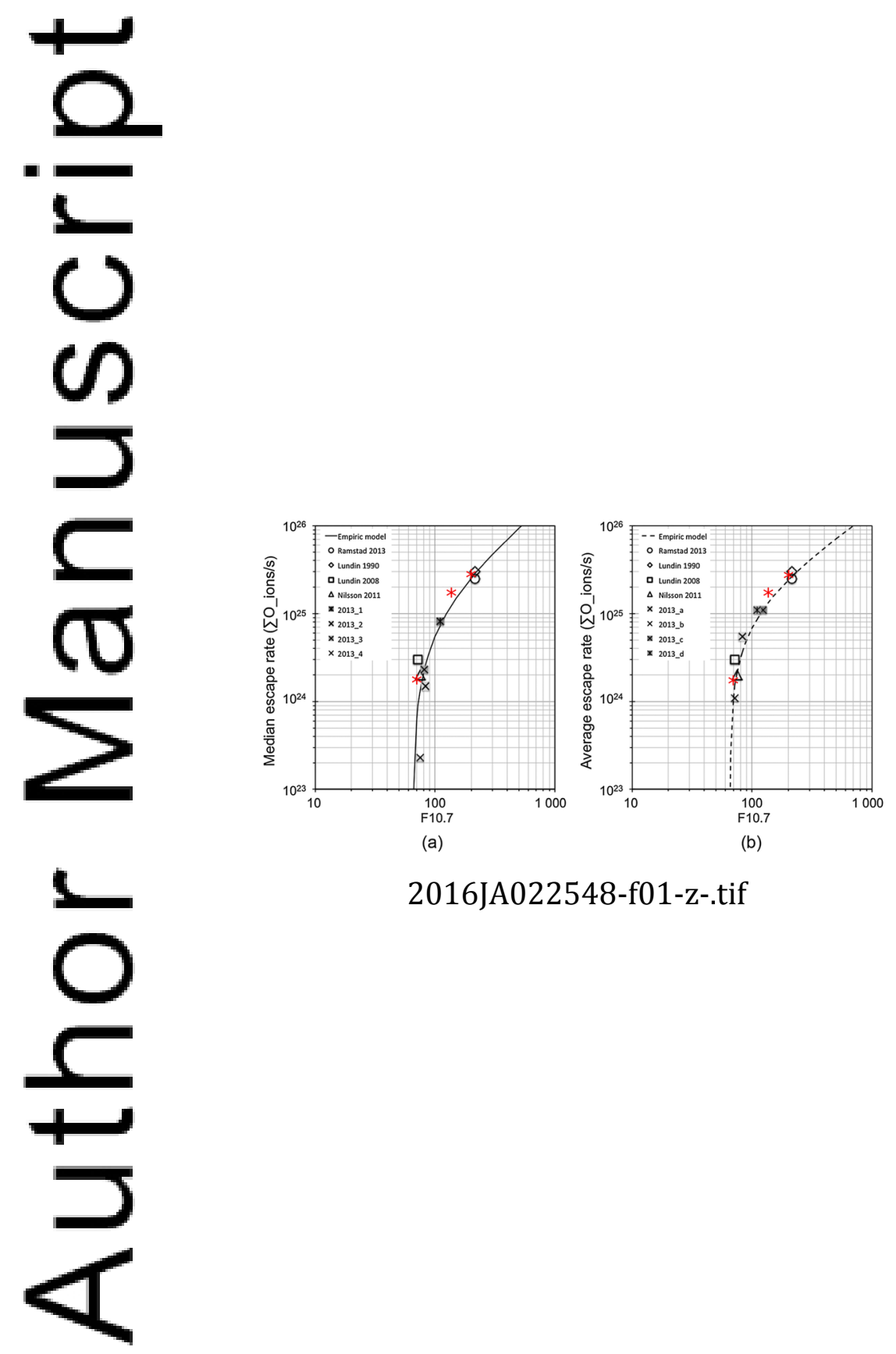

2016JA022548-f01-z-.tif

This article is protected by copyright. All rights reserved. 


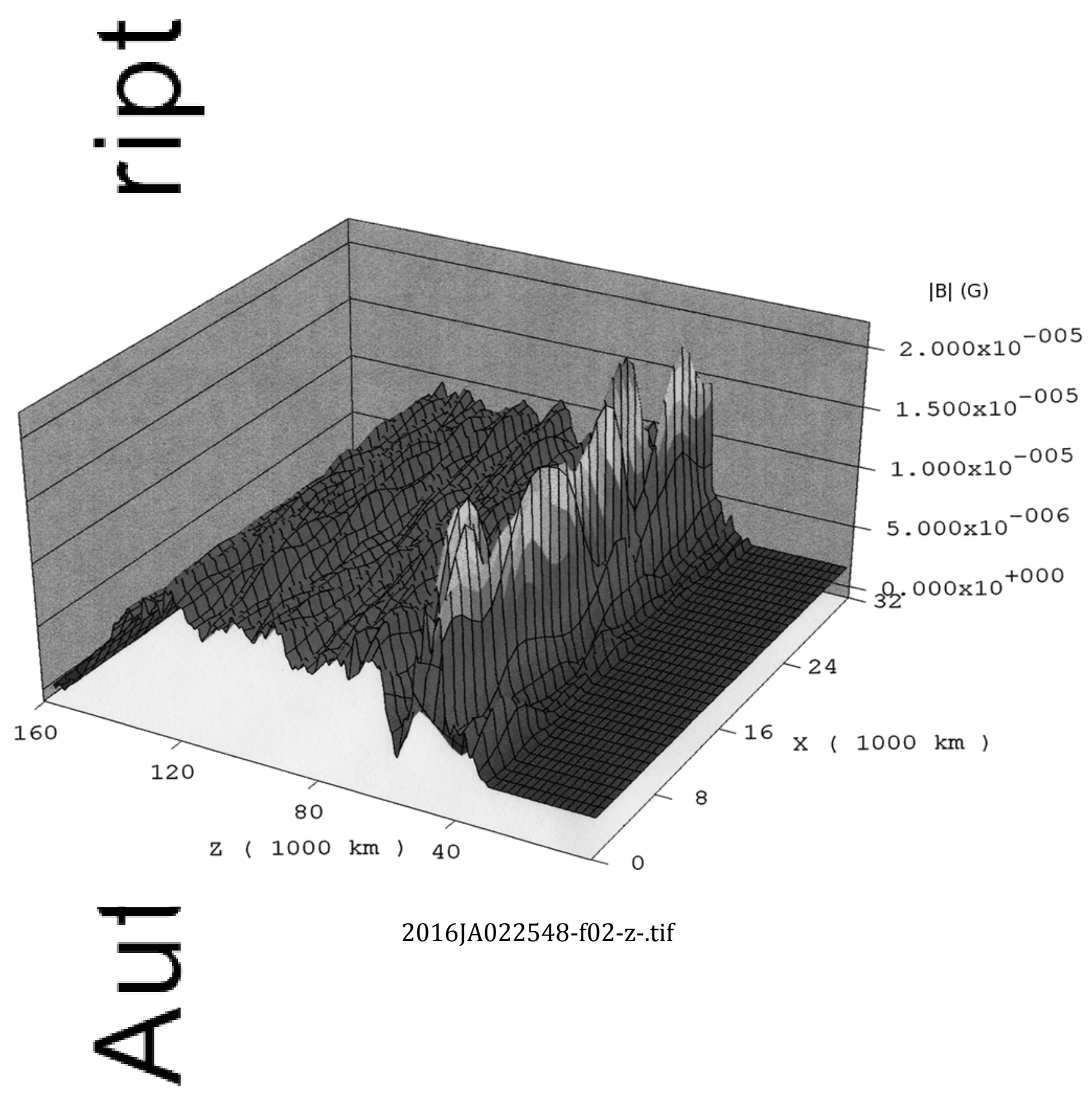

This article is protected by copyright. All rights reserved. 


\section{$\check{2}$}

Bagenal et al: Uranus Bow Shock
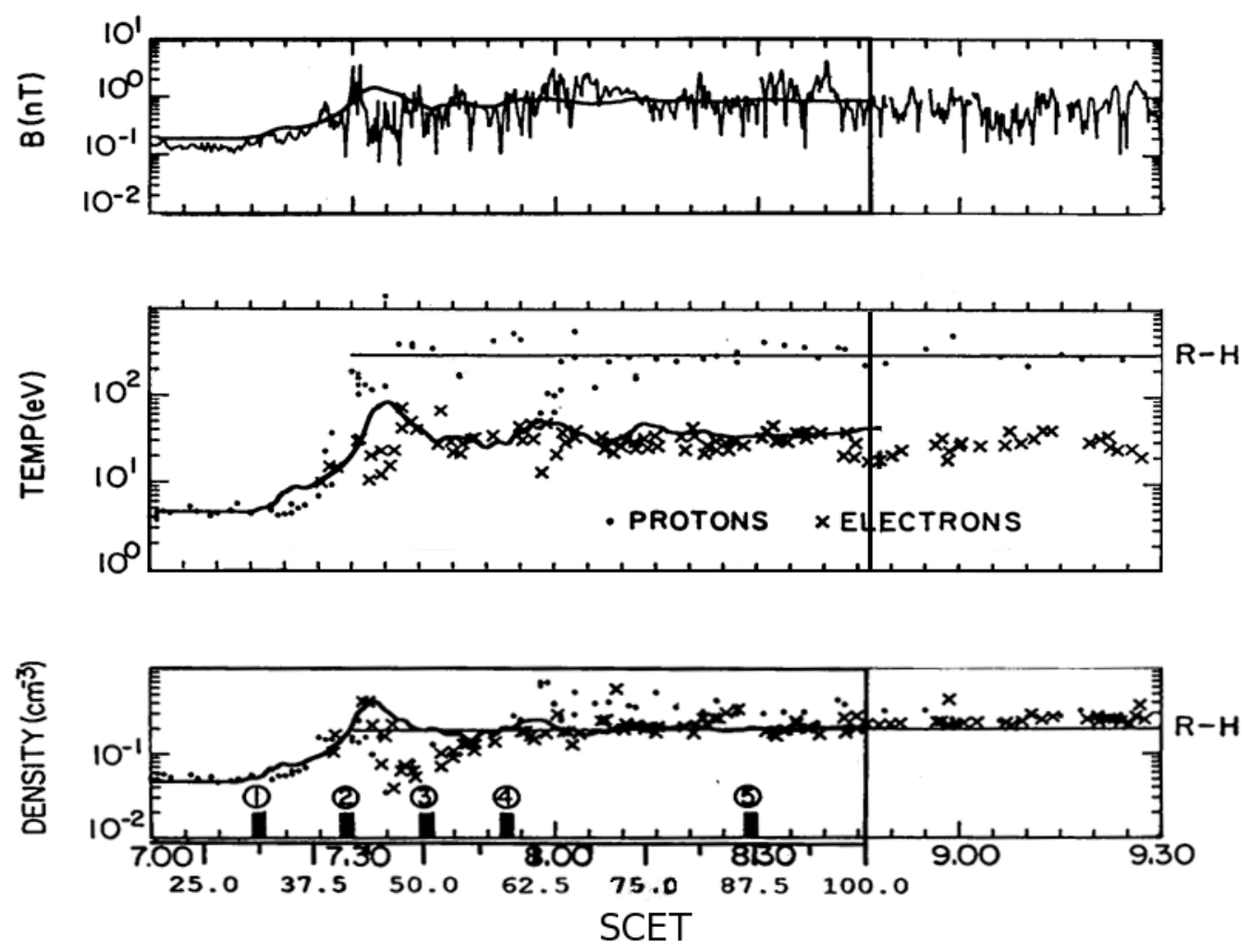

2016JA022548-f03-z-.tif

This article is protected by copyright. All rights reserved. 

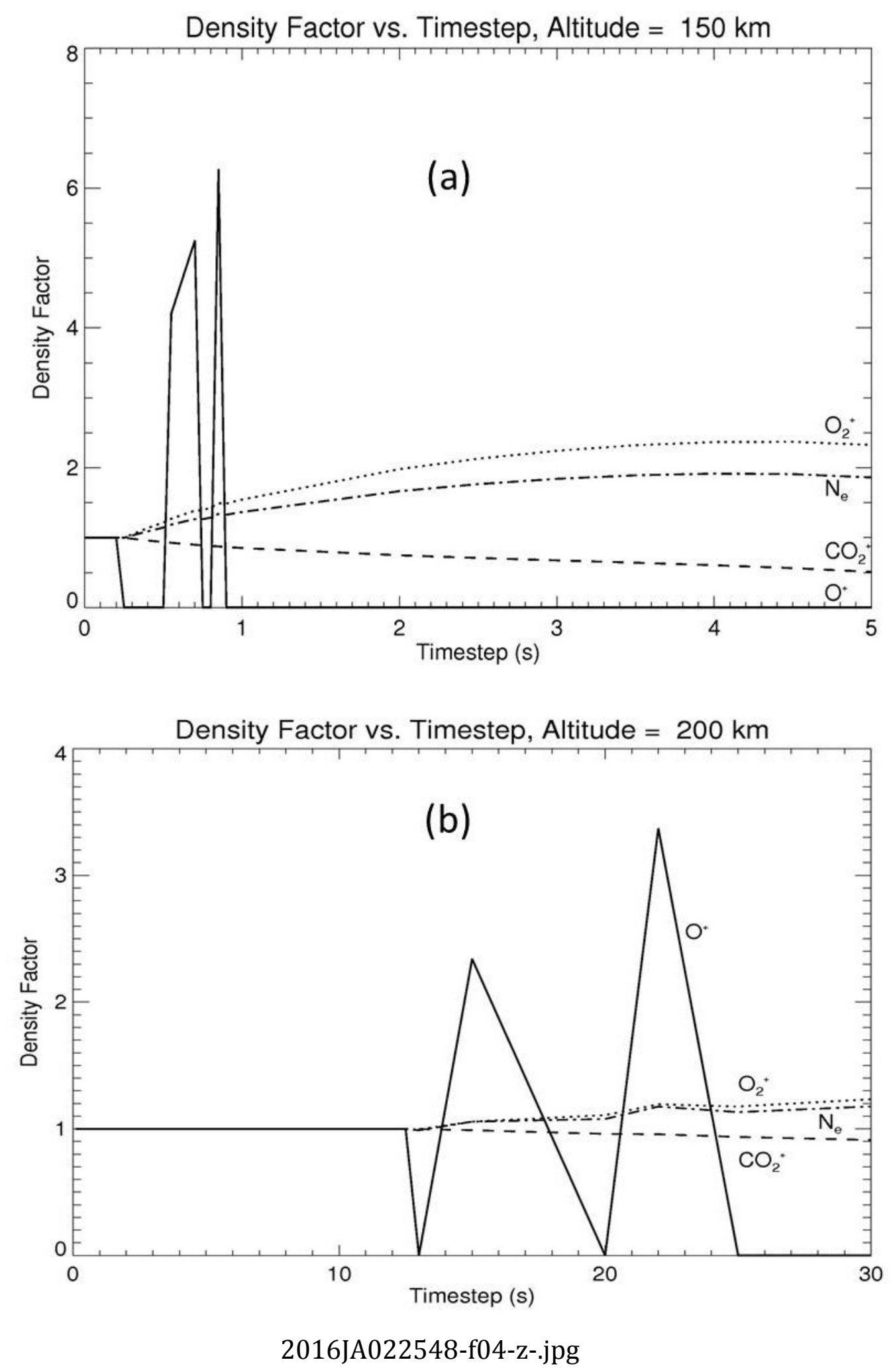

This article is protected by copyright. All rights reserved. 


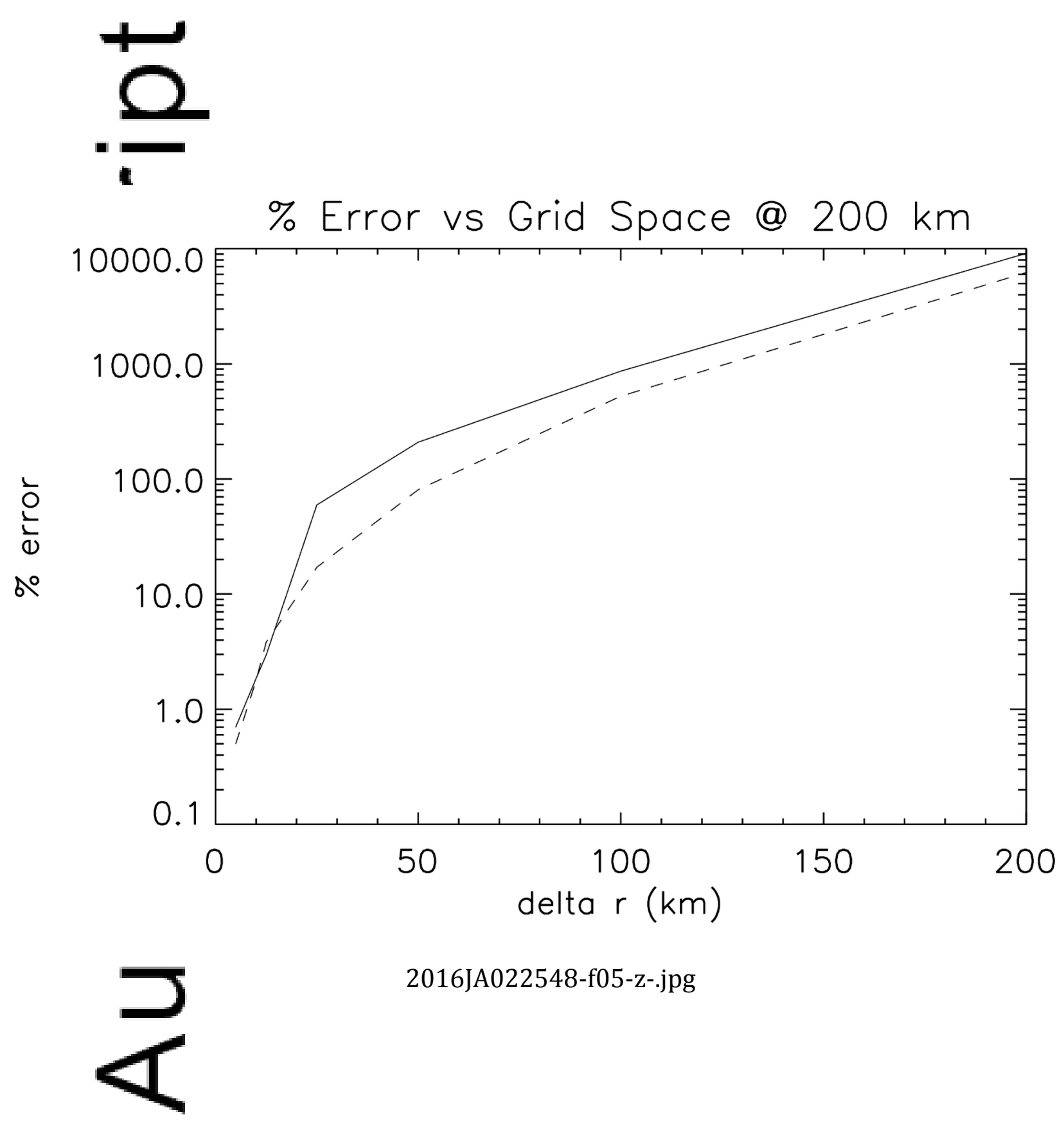

This article is protected by copyright. All rights reserved. 
NEUTRAL TEMPERATURE

$U T=0.00 \mathrm{HEIGHT}=200.00(\mathrm{KM})$

(a)
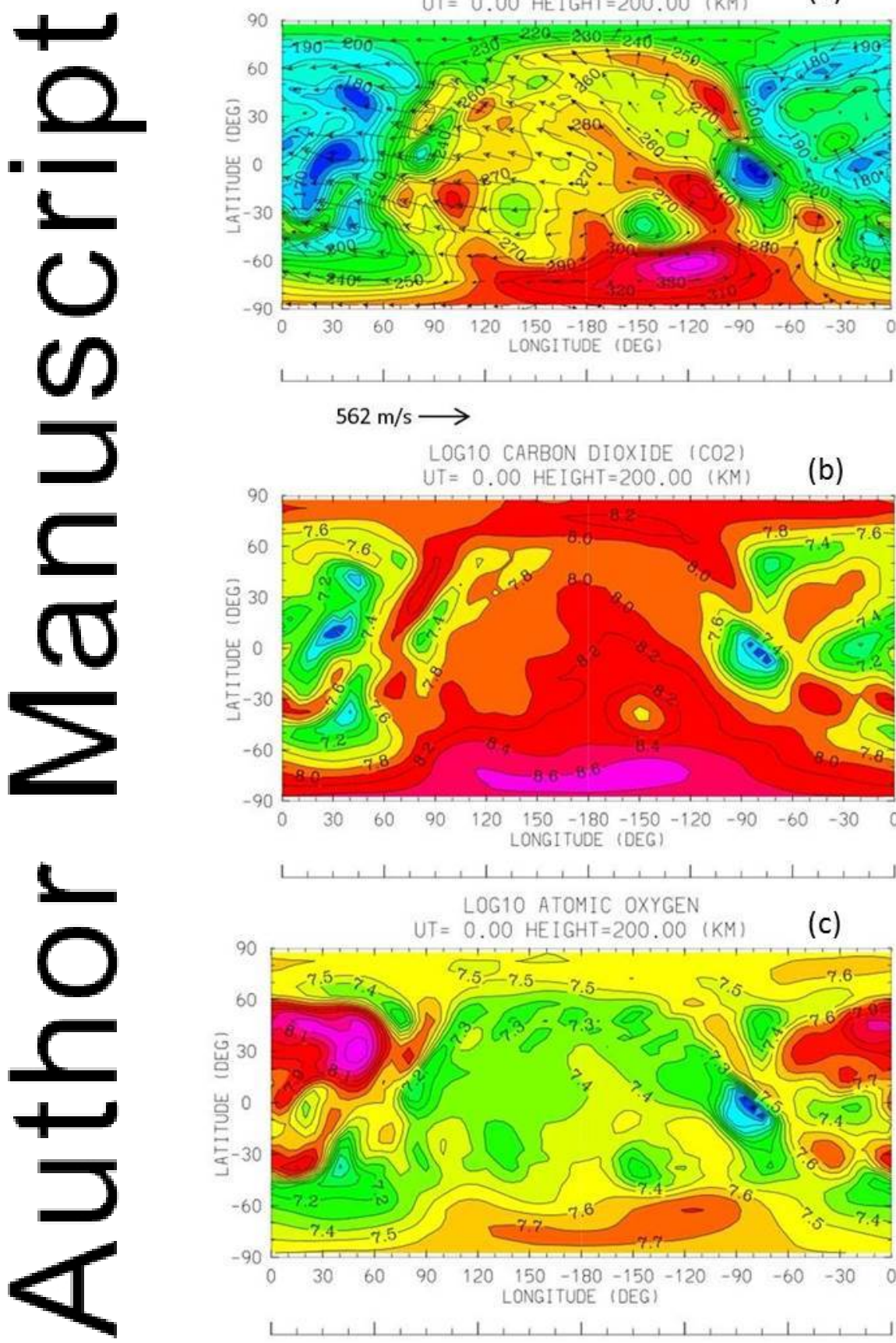

LOG10 CARBON DIOXIDE (CO2) $U T=0.00 \mathrm{HEIGHT}=200.00(\mathrm{KM})$

(b)

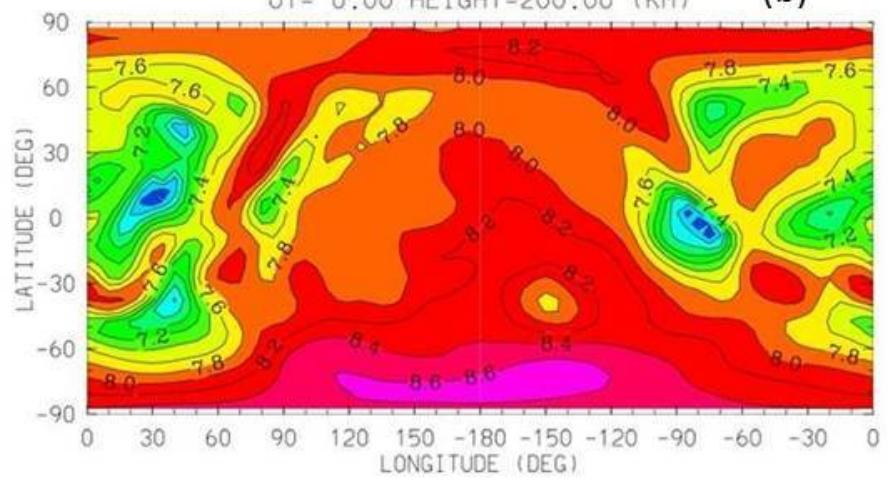

LOG10 ATOMIC OXYGEN $U T=0.00 \mathrm{HEIGHT}=200.00 \quad(\mathrm{KM})$

(c)

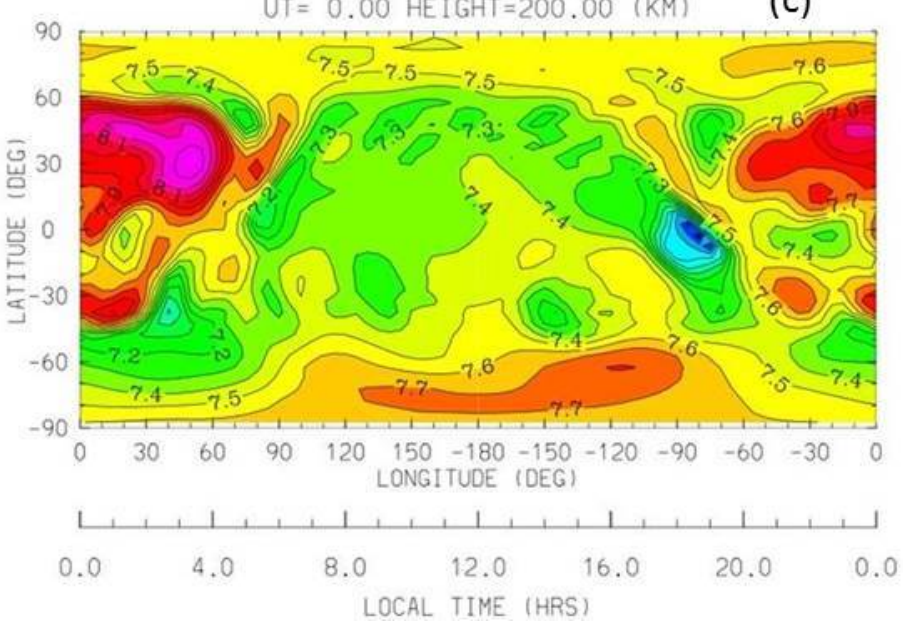

2016JA022548-f06-z-.jpg

This article is protected by copyright. All rights reserved. 

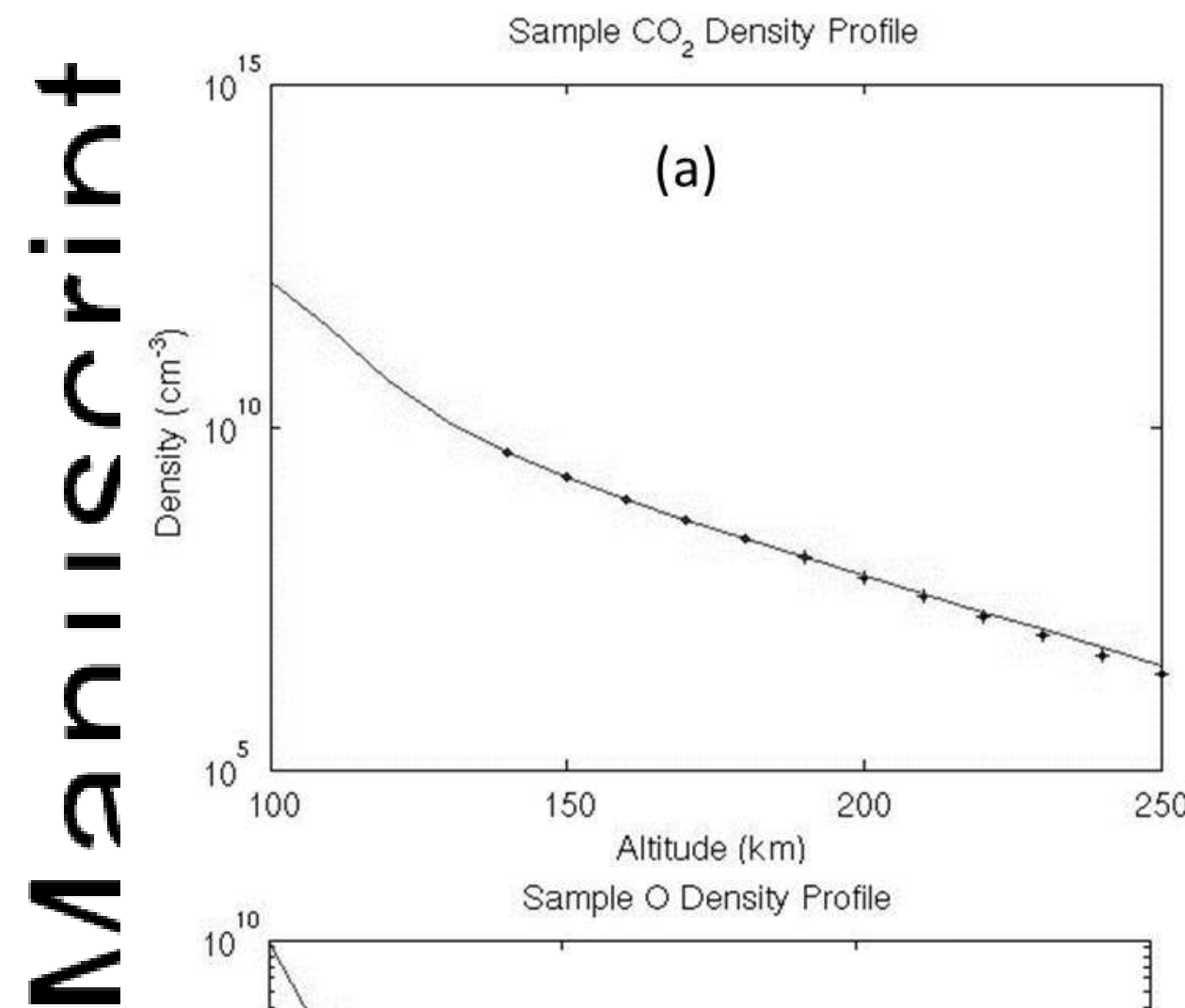

Sample O Density Profile

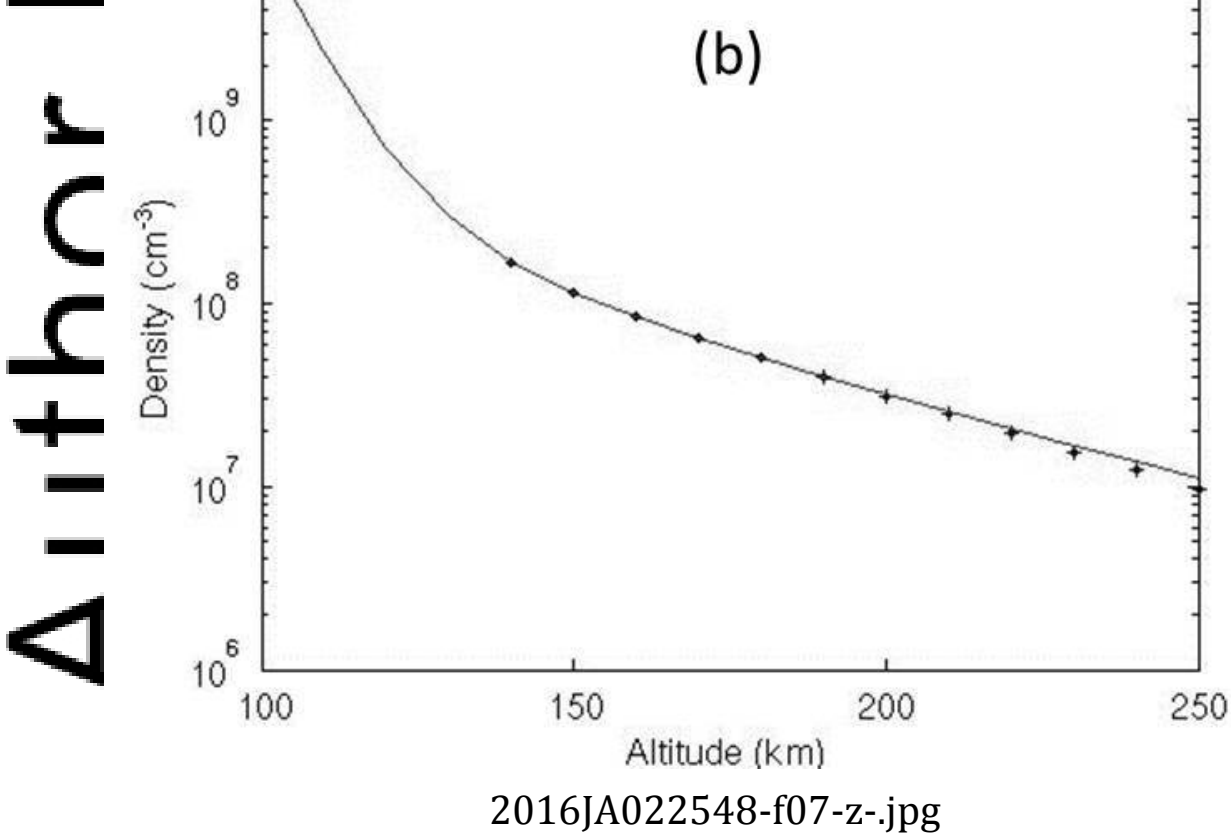

This article is protected by copyright. All rights reserved. 

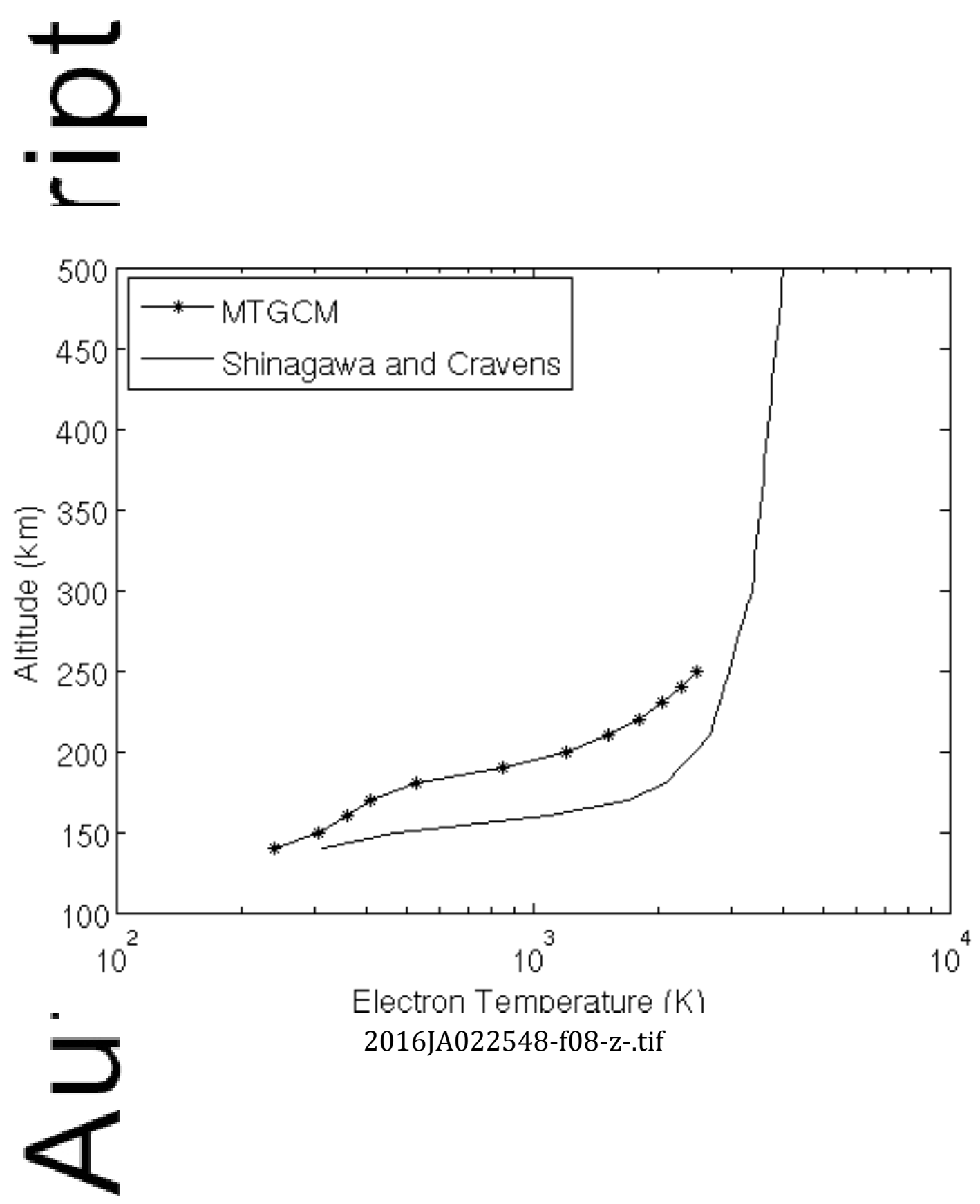

Electron Temperature ( $\mathrm{K}$ ) 2016JA022548-f08-z-.tif 


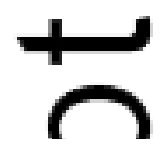

\section{$\log \left[\mathrm{O}_{2}^{+}\left(\mathrm{cm}^{-3}\right)\right]$}

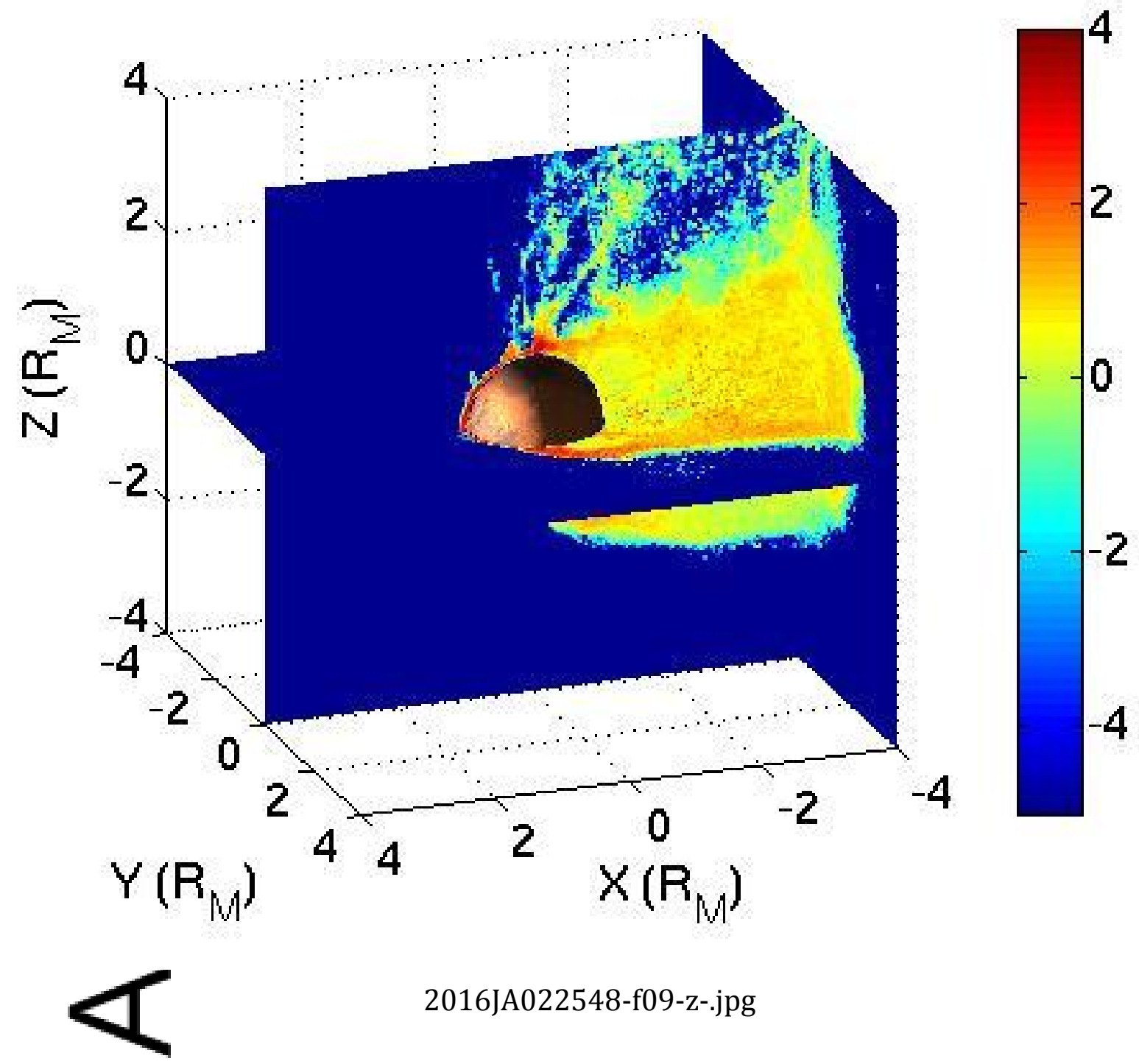



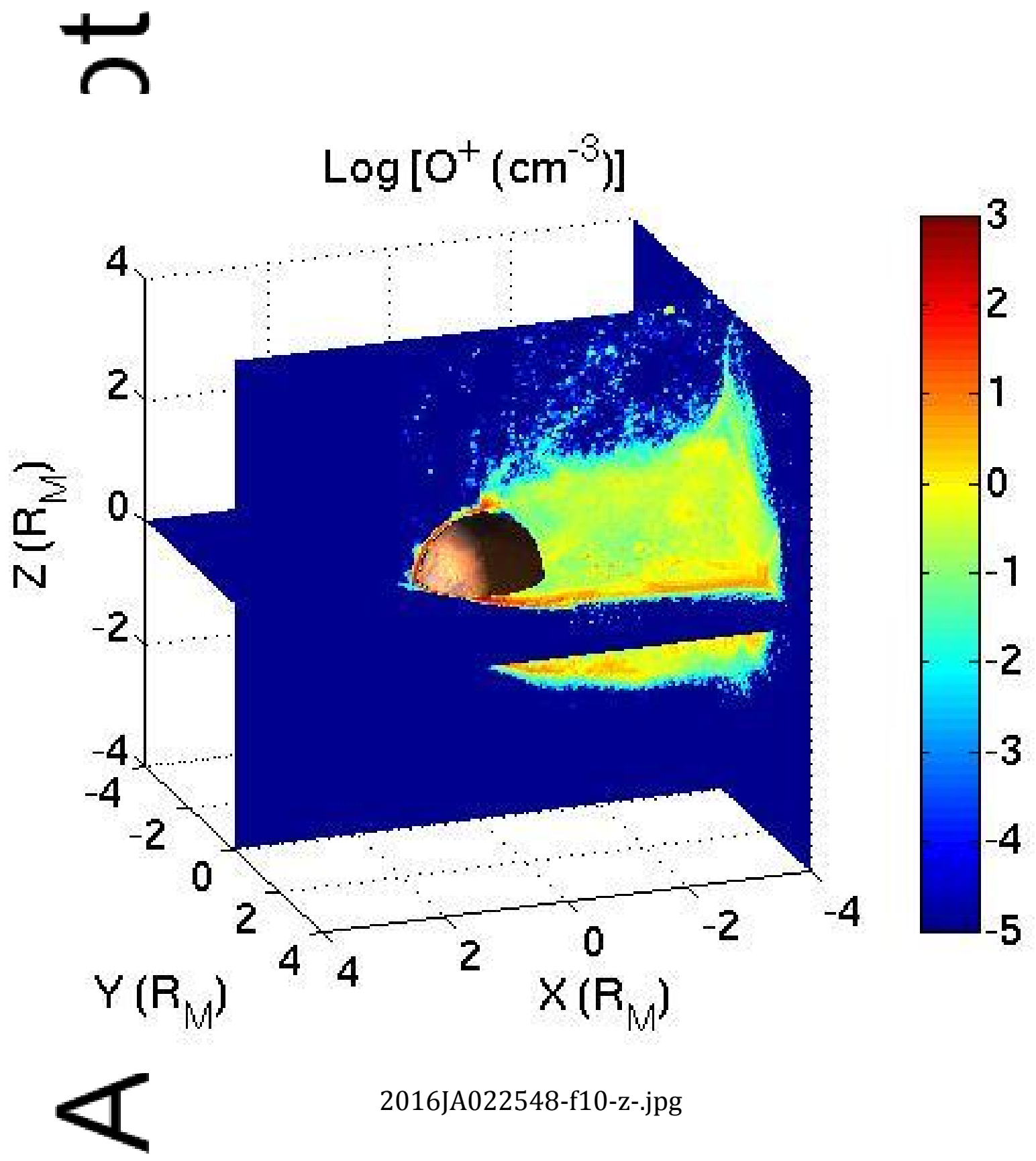

This article is protected by copyright. All rights reserved. 


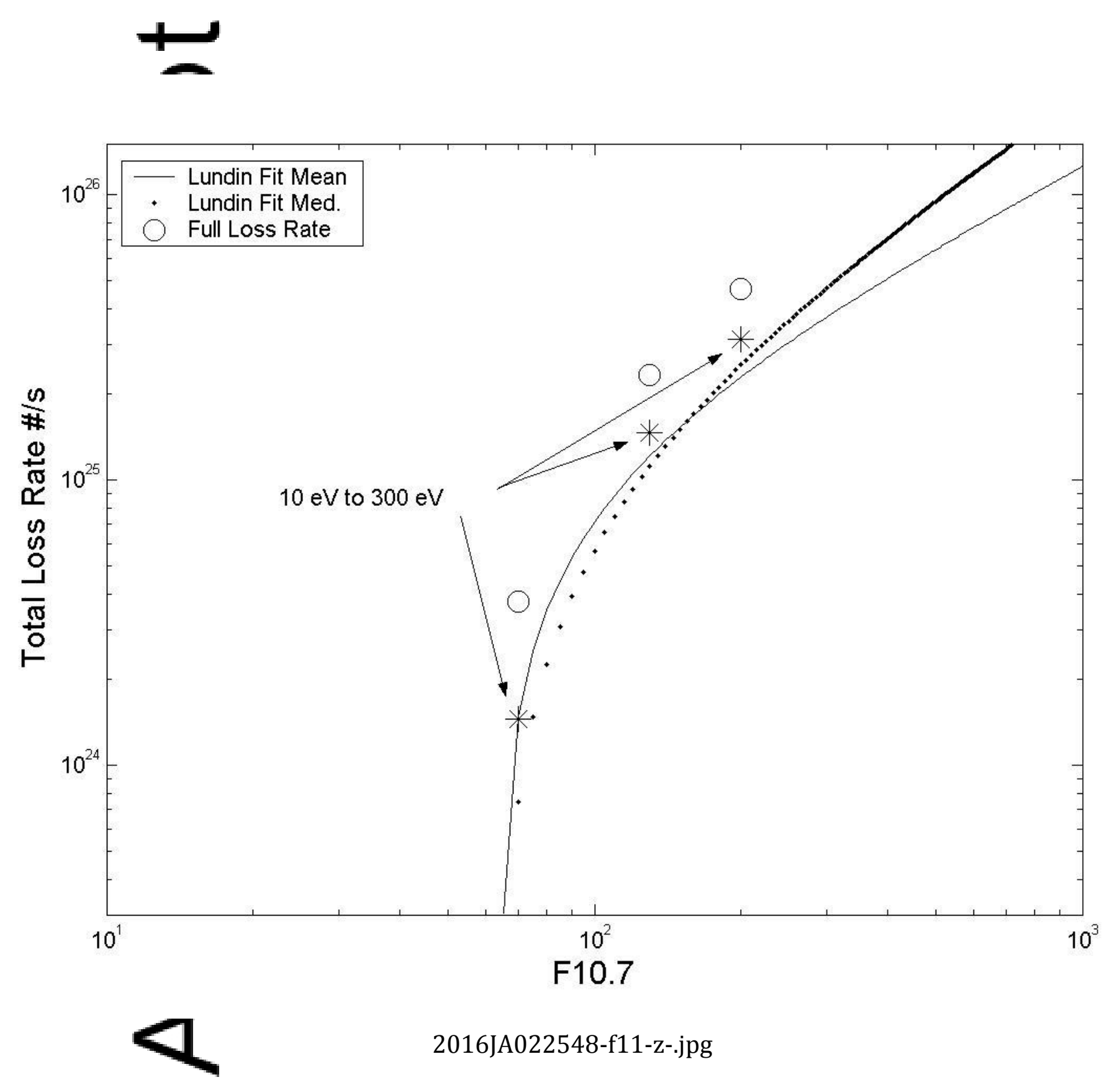

This article is protected by copyright. All rights reserved. 


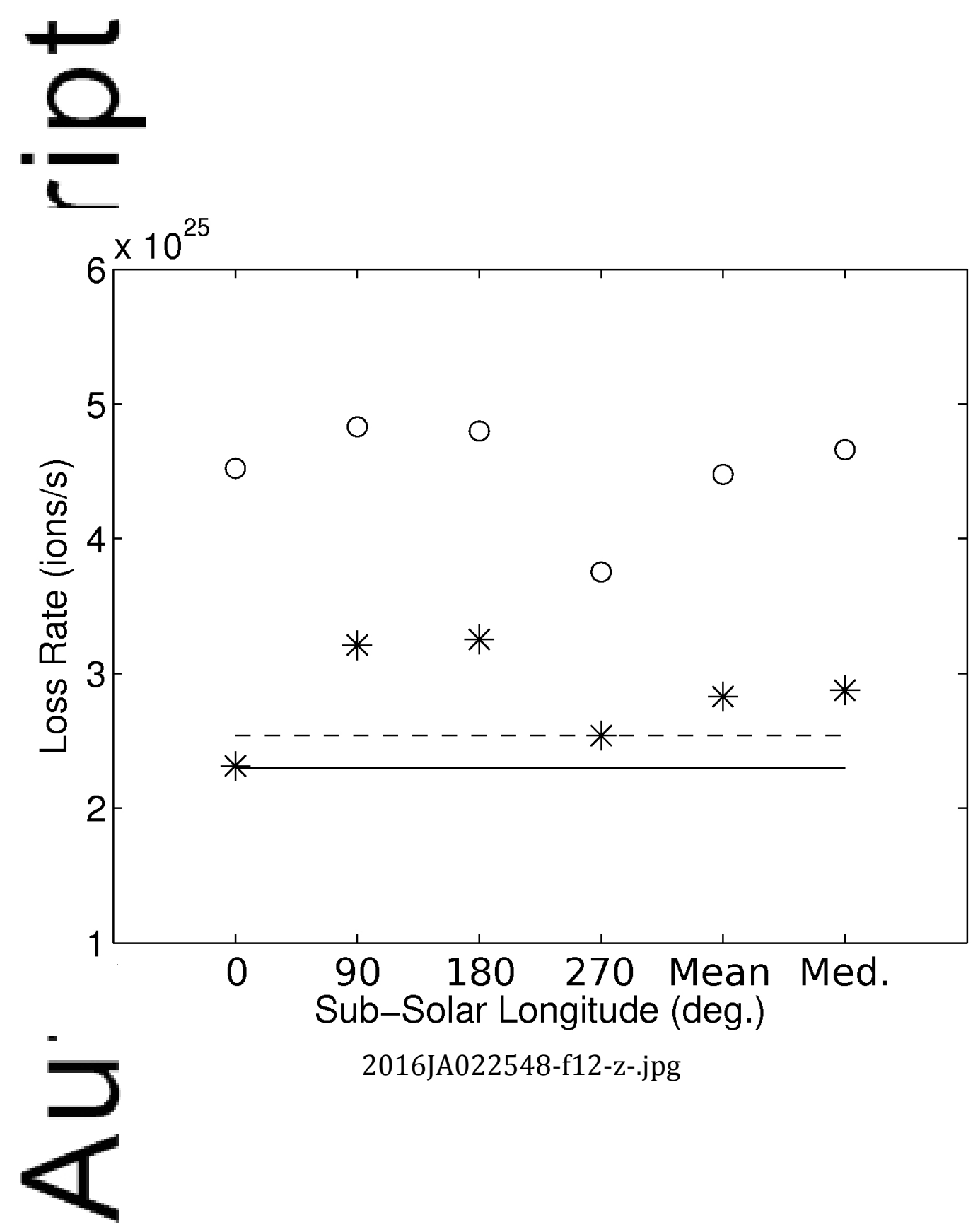

This article is protected by copyright. All rights reserved. 


\section{$\log \left[\mathrm{O}_{2}^{+} / \mathrm{O}^{+}\right.$Flux $]$}

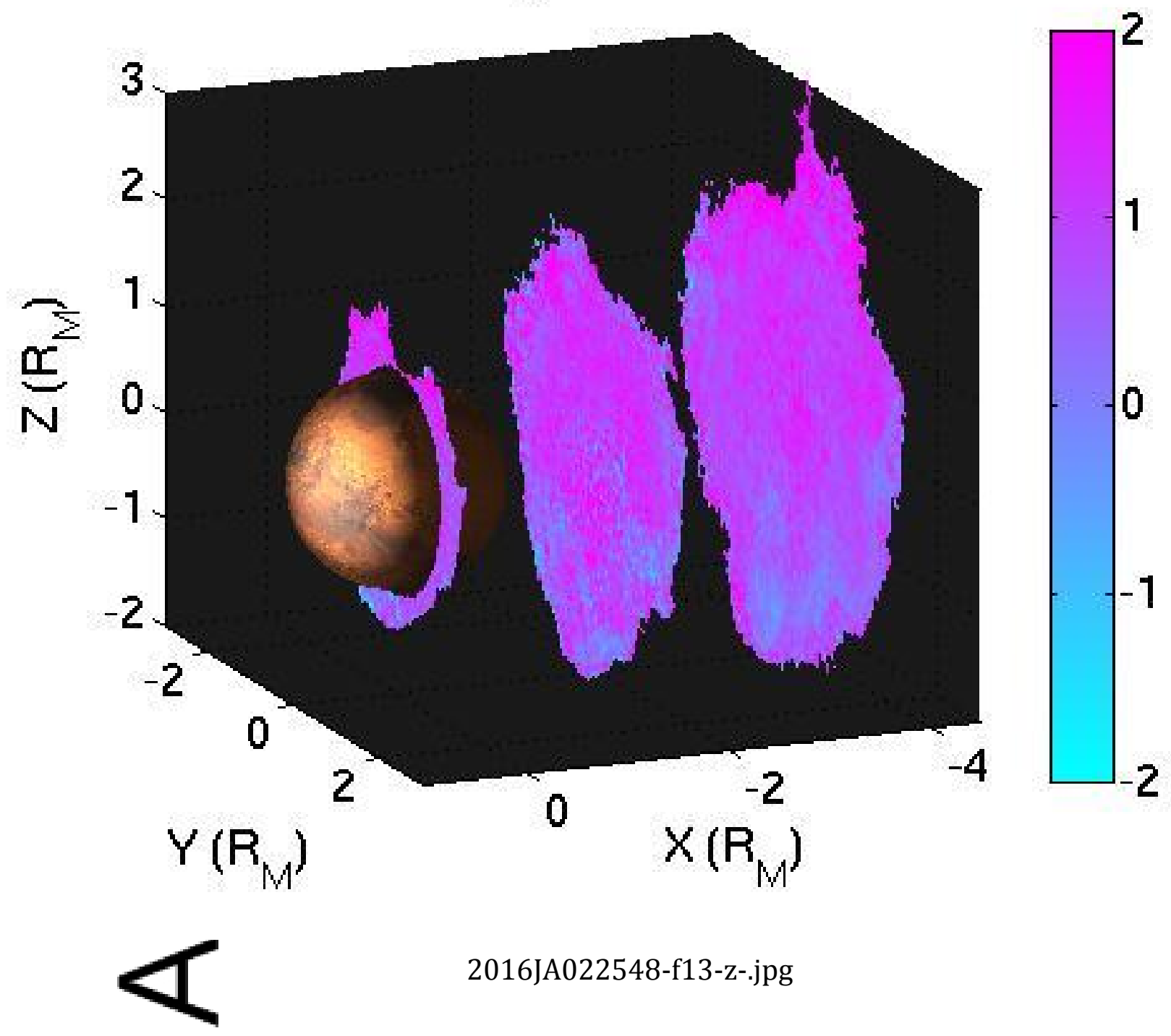




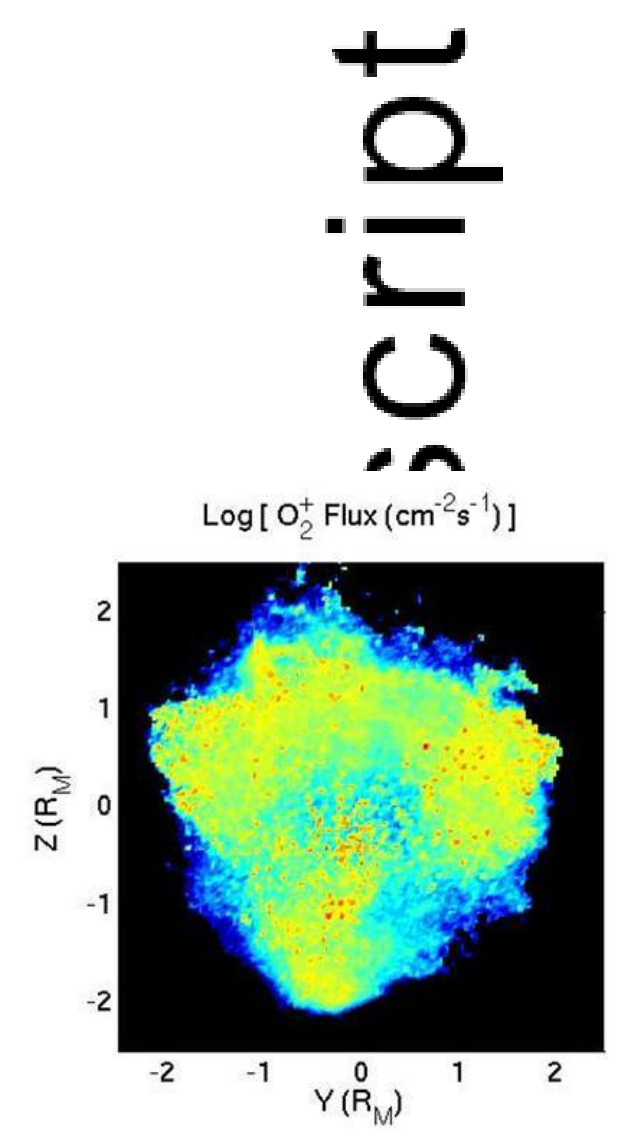

(a)

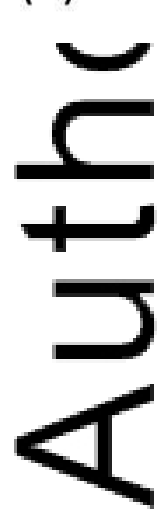

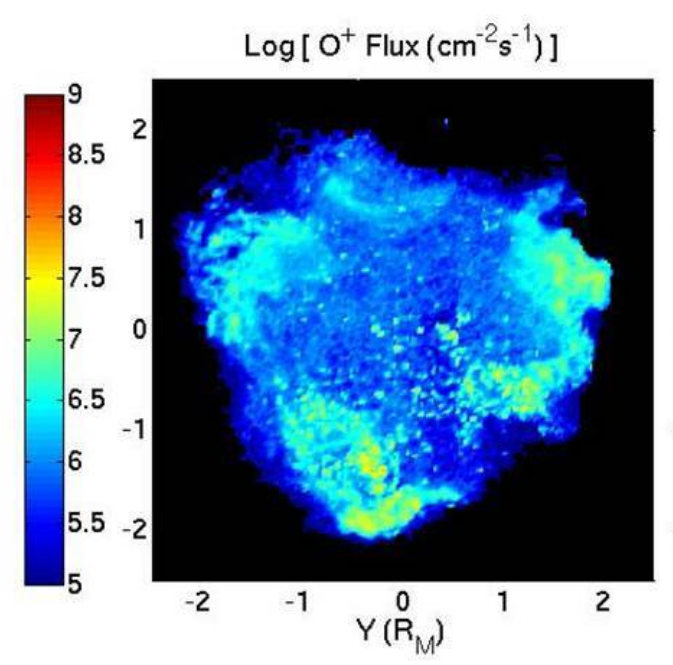

(b)

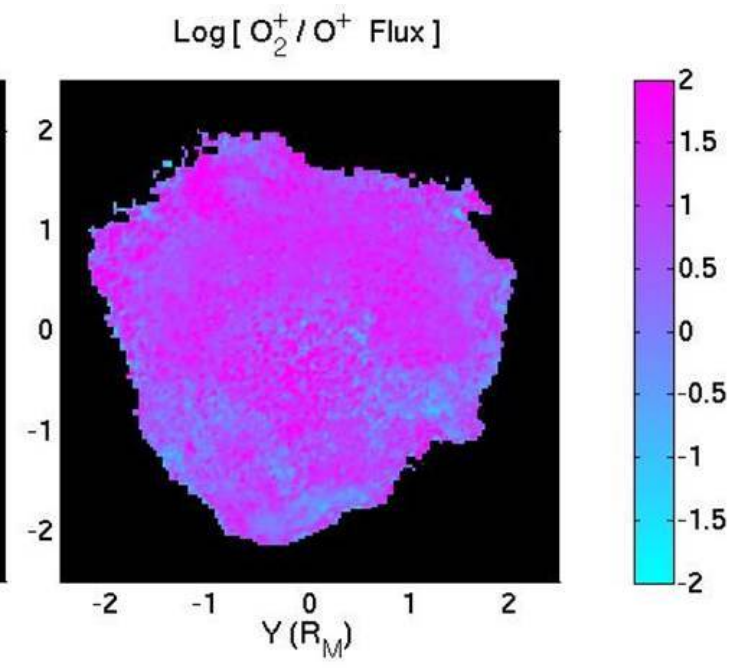

(c)

2016JA022548-f14-z-jpg

This article is protected by copyright. All rights reserved. 


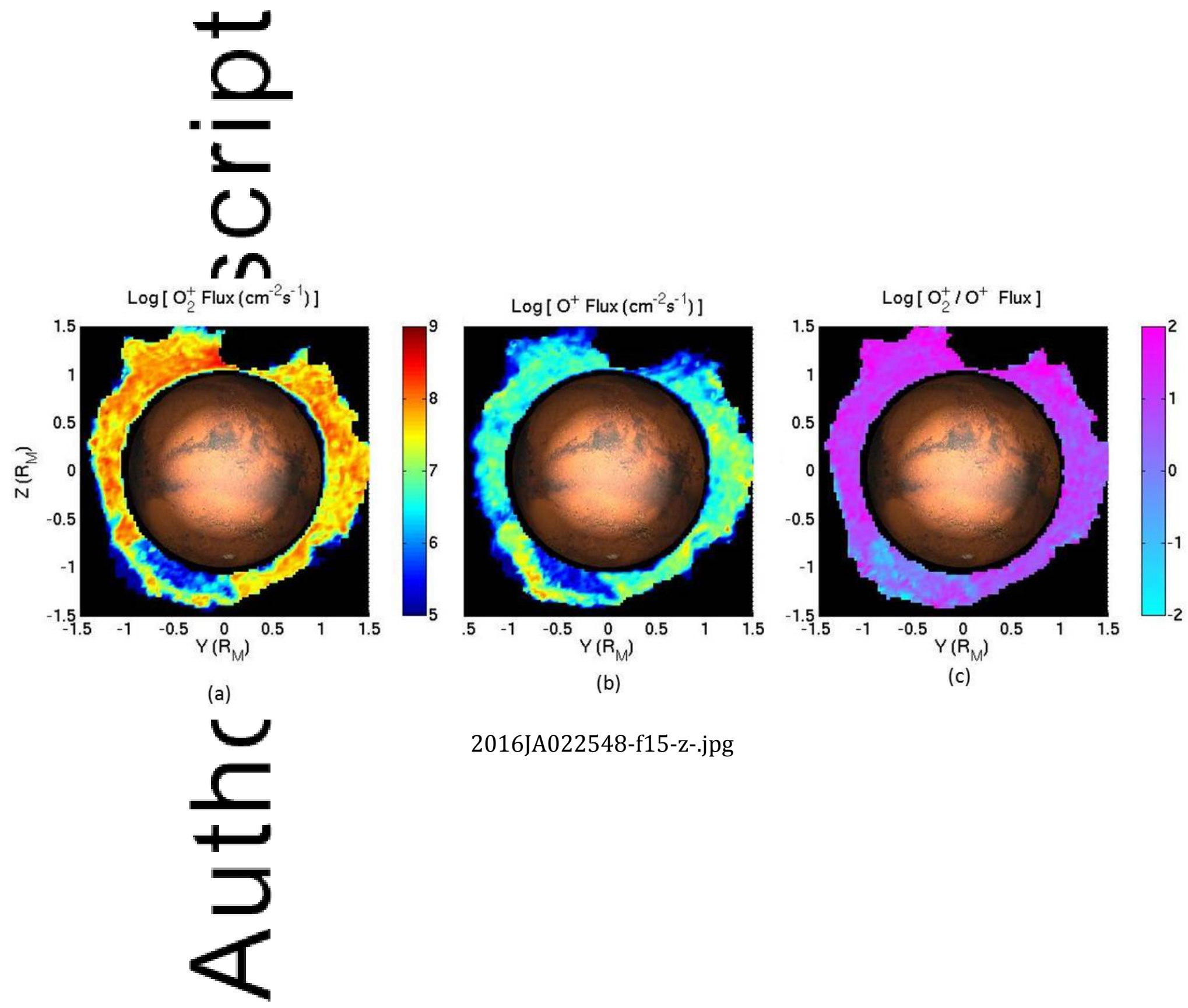

This article is protected by copyright. All rights reserved. 